\title{
Riemann boundary value problem for H-2-monogenic function in Hermitian Clifford analysis
}

\author{
Longfei Gu and Zunwei Fu*
}

*Correspondence:

zwfu@mail.bnu.edu.cn

Department of Mathematics, Linyi

University, Linyi, Shandong 276005, P.R. China

\section{空 Springer}

\begin{abstract}
Hermitian Clifford analysis has emerged as a new and successful branch of Clifford analysis, offering yet a refinement of the Euclidean case; it focuses on the simultaneous null solutions of two Hermitian Dirac operators. Using a circulant matrix approach, we will study the $R_{0}$ Riemann type problems in Hermitian Clifford analysis. We prove a mean value formula for the Hermitian monogenic function. We obtain a Liouville-type theorem and a maximum module for the function above. Applying the Plemelj formula, integral representation formulas, and a Liouville-type theorem, we prove that the $R_{0}$ Riemann type problems for Hermitian monogenic and Hermitian-2-monogenic functions are solvable. Explicit representation formulas of the solutions are also given.
\end{abstract}

Keywords: Hermitian Clifford analysis; Riemann type problems; Hermitian monogenic function

\section{Introduction}

The classical Riemann boundary value problem (BVP for short) theory in the complex plane has been systematically developed, see [1] and [2]. It is natural to generalize the classical Riemann BVP theory to higher dimensions. Euclidean Clifford analysis is a higher dimensional function theory offering a refinement of classical harmonic analysis and a generation of complex in plane analysis. The theory is centered around the concept of monogenic functions, see [3-6], etc. Under the framework, in [7-12], many interesting results about BVP for monogenic functions in Clifford analysis were presented. In [13] and [14], Riemann BVP for harmonic functions (i.e., 2-monogenic functions) and biharmonic functions were studied, the solutions are given in an explicit way.

More recently, Hermitian Clifford analysis has emerged as a new and successful branch of Clifford analysis, offering yet a refinement of the Euclidean case; it focuses on the simultaneous null solutions of two Hermitian Dirac operators invariant under the action of the unitary group. This function theory can be found in [15] and [16], etc. In [17], based on the complex Clifford algebra $\mathbb{C}_{2 n}$, the Hermitian Cauchy integral formulas were constructed in the framework of circulant $(2 \times 2)$ matrix functions, and the intimate relationship with holomorphic function theory of several complex variables was considered. For details, we refer to [17-20]. In [18] and [21], a matrix Hilbert transform in Hermitian Clifford analysis was studied, and analogs of characteristic properties of the matrix Hilbert trans-

○2014 Gu and Fu; licensee Springer. This is an Open Access article distributed under the terms of the Creative Commons Attribution License (http://creativecommons.org/licenses/by/2.0), which permits unrestricted use, distribution, and reproduction in any medium, provided the original work is properly cited. 
form in classical analysis and orthogonal Clifford analysis were given, for example by the usual Plemelj-Sokhotski formula. Under this setting it is natural to consider the Riemann BVP. In [22], the Riemann BVP for (left) Helmholtz H-monogenic functions (i.e., null solutions of perturbed Hermitian Dirac operators in the framework of Hermitian Clifford analysis). If the perturbed value vanishes, $\mathcal{D}_{\left(\underline{Z}, \underline{Z}^{\dagger}\right)}^{\mathcal{K}}$ is $\mathcal{D}_{\left(\underline{Z}, \underline{Z}^{\dagger}\right)}$, then the $R_{-1}$ Riemann BVP for H-monogenic circulant $(2 \times 2)$ matrix functions was solved. Also, we naturally consider $R_{0}$ Riemann BVP for $\mathbf{H}$-monogenic circulant $(2 \times 2)$ matrix functions (i.e., null solutions to $\left.\mathcal{D}_{\left(\underline{Z}, \underline{Z}^{\dagger}\right)}\right)$ and $\mathbf{H}$-2-monogenic circulant $(2 \times 2)$ matrix functions (i.e., null solutions to $\left.\mathcal{D}_{\left(\underline{Z}, \underline{Z}^{\dagger}\right)}^{2}\right)$. Roughly speaking $R_{0}$ Riemann BVP means that we prescribe that the solutions are bounded at infinity. Up to present, as far as we know, it is a new problem. In this paper, motivated by $[8,9,13,14,17,18]$, we will consider $R_{0}$ Riemann BVP for H-2-monogenic circulant $(2 \times 2)$ matrix functions in Hermitian Clifford analysis. Applying the integral representation formulas of $\mathbf{H}$-monogenic circulant $(2 \times 2)$ matrix functions and $\mathbf{H}-2$ monogenic circulant $(2 \times 2)$ matrix functions, we get mean values formulas. Furthermore we prove a maximum modulus theorem and a Liouville theorem in Hermitian Clifford analysis. Finally we get explicit solutions for $R_{0}$ Riemann BVP for H-2-monogenic circulant $(2 \times 2)$ matrix functions in Hermitian Clifford analysis. Some results of [14] and [22] are generalized in our paper.

\section{Preliminaries}

In this section we recall some basic facts about Clifford algebras and Hermitian Clifford analysis which will be needed in the sequel. More details can also be found in [4] and [5].

Let $V_{2 n, 0}$ be an $2 n$-dimensional $(n \geq 1)$ real linear space with basis $\left\{e_{1}, e_{2}, \ldots, e_{2 n}\right\}$, $\mathrm{Cl}\left(V_{2 n, 0}\right)$ be the $2^{2 n}$-dimensional real linear space with basis

$$
\left\{e_{A}, A=\left\{h_{1}, \ldots, h_{r}\right\} \in \mathcal{P} N, 1 \leq h_{1}<\cdots<h_{r} \leq 2 n\right\},
$$

where $N$ stands for the set $\{1, \ldots, 2 n\}$ and let $\mathcal{P} N$ denote the family of all order-preserving subsets of $N$ in the above way. Now denote $e_{\emptyset}$ by $e_{0}$ and $e_{h_{1} \cdots h_{r}}$ by $e_{A}$ for $A=\left\{h_{1}, \ldots, h_{r}\right\} \in$ $\mathcal{P} N$. The product on $\mathrm{Cl}\left(V_{2 n, 0}\right)$ is defined by

$$
\begin{cases}e_{A} e_{B}=(-1)^{\#(A \cap B)}(-1)^{P(A, B)} e_{A \triangle B}, & \text { if } A, B \in \mathcal{P} N, \\ \lambda \mu=\sum_{A, B \in \mathcal{P} N} \lambda_{A} \mu_{B} e_{A} e_{B}, & \text { if } \lambda=\sum_{A \in \mathcal{P} N} \lambda_{A} e_{A}, \mu=\sum_{B \in \mathcal{P}_{N}} \mu_{B} e_{B},\end{cases}
$$

where \# $(A)$ is the cardinal number of the set $A$, the number $P(A, B)=\sum_{j \in B} P(A, j), P(A, j)=$ $\#\{i, i \in A, i>j\}$, the symmetric difference set $A \triangle B$ is also order-preserving in the above way, and $\lambda_{A} \in \mathbf{R}$ is the coefficient of the $e_{A}$-component of the Clifford number $\lambda$. Also, denote $[\lambda]_{A}$ by $\lambda_{A}$. It follows at once from the multiplication rule (2.1) that $e_{0}$ is the identity element written now as 1 and, in particular,

$$
\begin{cases}e_{i}^{2}=-1, & \text { if } i=1, \ldots, 2 n, \\ e_{i} e_{j}=-e_{j} e_{i}, & \text { if } 1 \leq i<j \leq 2 n, \\ e_{h_{1}} e_{h_{2}} \ldots e_{h_{r}}=e_{h_{1} h_{2} \ldots h_{r}}, & \text { if } 1 \leq h_{1}<h_{2}<\cdots<h_{r} \leq 2 n\end{cases}
$$

Thus $\mathrm{Cl}\left(V_{2 n, 0}\right)$ is a real linear, associative, but non-commutative algebra and it is called the Clifford algebra over $V_{2 n, 0}$. An involution is defined by

$$
\begin{cases}\bar{e}_{A}=(-1)^{\frac{\#(A)(\#(A)+1)}{2}} e_{A}, & \text { if } A \in \mathcal{P} N, \\ \bar{\lambda}=\sum_{A \in \mathcal{P} N} \lambda_{A} \bar{e}_{A}, & \text { if } \lambda=\sum_{A \in \mathcal{P} N} \lambda_{A} e_{A} .\end{cases}
$$


From (2.1) and (2.3), we have

$$
\begin{cases}\bar{e}_{i}=-e_{i}, & \text { if } i=1, \ldots, 2 n \\ \overline{\lambda \mu}=\bar{\mu} \bar{\lambda}, & \text { for any } \lambda, \mu \in \mathrm{Cl}\left(V_{2 n}, 0\right)\end{cases}
$$

The Euclidean space $\mathbf{R}^{2 n}$ is embedded in $\mathrm{Cl}\left(V_{2 n, 0}\right)$ by identifying $\left(X_{1}, \ldots, X_{2 n}\right)$ with the Clifford vector $\underline{X}$ given by

$$
\underline{X}=\sum_{j=1}^{2 n} e_{j} X_{j}
$$

Note that the square of $\underline{X}$ is scalar valued and equals the norm squared up to a minus sign: $\underline{X}^{2}=-\langle\underline{X}, \underline{X}\rangle=-|\underline{X}|^{2}$. The dual of $\underline{X}$ is the vector-valued first order differential operator

$$
\partial_{\underline{X}}=\sum_{j=1}^{2 n} e_{j} \partial_{X_{j}}
$$

called a Dirac operator. It is precisely this Dirac operator which underlies the notion of monogenicity of a function, a notion which is the higher dimensional counterpart of holomorphy in the complex plane. A function $f$ defined and differentiable in an open region $\Omega$ of $\mathbf{R}^{2 n}$ and taking values in $\mathrm{Cl}\left(V_{2 n, 0}\right)$ is called (left) monogenic in $\Omega$ if $\partial_{\underline{X}}[f]=0$. As the Dirac operator factorizes the Laplacian, $\Delta_{2 n}=-\partial_{\underline{X}}^{2}$, monogenicity can be regarded as a refinement of harmonicity. We refer to this setting as the orthogonal case, since the fundamental group leaving the Dirac operator $\partial_{\underline{X}}$ invariant is the special orthogonal group $S O(2 n, \mathbf{R})$, which is doubly covered by the $\operatorname{Spin}(m)$ group of the Clifford algebra $\mathrm{Cl}\left(V_{2 n, 0}\right)$. For this reason, the Dirac operator is also called rotation invariant. When allowing for complex constants, the set of generators $\left\{e_{1}, \ldots, e_{2 n}\right\}$ produces the complex Clifford algebra $\mathbb{C}_{2 n}$, being the complexification of the real Clifford algebra $\mathrm{Cl}\left(V_{2 n, 0}\right)$, i.e. $\mathbb{C}_{2 n}=\mathrm{Cl}\left(V_{2 n, 0}\right) \oplus i \mathrm{Cl}\left(V_{2 n, 0}\right)$. Any complex Clifford number $\lambda \in \mathbb{C}_{2 n}$ may be written as $\lambda=a+i b, a, b \in \mathrm{Cl}\left(V_{2 n, 0}\right)$, an observation leading to the definition of the Hermitian conjugation $\lambda^{\dagger}=(a+i b)^{\dagger}=\bar{a}-i \bar{b}$, where the bar notation stands for the usual Clifford conjugation in $\operatorname{Cl}\left(V_{2 n, 0}\right)$, i.e. the main anti-involution for which $\bar{e}_{j}=-e_{j}, j=1, \ldots, 2 n$. This Hermitian conjugation also leads to a Hermitian inner product and its associated norm on $\mathbb{C}_{2 n}$ is given by $(\lambda, \mu)=\left[\lambda^{\dagger} \mu\right]_{0}$ and $|\lambda|=\sqrt{\left[\lambda^{\dagger} \lambda\right]_{0}}=\left(\sum_{A}\left|\lambda_{A}\right|^{2}\right)^{\frac{1}{2}}$.

The above will be the framework for the so-called Hermitian Clifford analysis, yet a refinement of orthogonal Clifford analysis. An elegant way for introducing this setting consists in considering a so-called complex structure, i.e. a specific $S O(2 n ; \mathbf{R})$-element $J$ for which $J^{2}=-\mathbf{1}$ (see [15-17]). Here, $J$ is chosen to act upon the generators $e_{1}, \ldots, e_{2 n}$ of the Clifford algebra as

$$
J\left[e_{j}\right]=-e_{n+j} \quad \text { and } \quad J\left[e_{n+j}\right]=e_{j}, \quad j=1, \ldots, n .
$$

With $J$ one may associate two projection operators $\frac{1}{2}(\mathbf{1} \pm i J)$ which will produce the main protagonists of the Hermitian setting by acting upon the corresponding objects in the orthogonal framework. First of all, the so-called Witt basis elements $\left\{f_{j}, f_{j}^{\dagger} \mid j=1,2, \ldots, n\right\}$ 
for the complex Clifford algebra $\mathbb{C}_{2 n}$ are obtained through the action of $\pm \frac{1}{2}(1 \pm i J)$ on the orthogonal basis elements $e_{j}$ :

$$
\begin{aligned}
& f_{j}=\frac{1}{2}(\mathbf{1}+i J)\left[e_{j}\right]=\frac{1}{2}\left(e_{j}-i e_{n+j}\right), \quad j=1, \ldots, n, \\
& f_{j}^{\dagger}=-\frac{1}{2}(\mathbf{1}-i J)\left[e_{j}\right]=-\frac{1}{2}\left(e_{j}+i e_{n+j}\right), \quad j=1, \ldots, n .
\end{aligned}
$$

These Witt basis elements satisfy the Grassmann identities,

$$
f_{j} f_{k}+f_{k} f_{j}=f_{j}^{\dagger} f_{k}^{\dagger}+f_{k}^{\dagger} f_{j}^{\dagger}=0, \quad j, k=1, \ldots, n,
$$

and the duality identities,

$$
f_{j} f_{k}^{\dagger}+f_{k}^{\dagger} f_{j}=\delta_{j k}, \quad j, k=1, \ldots, n
$$

Next we identify a vector $\underline{X}=\left(\underline{X}_{1}, \ldots, \underline{X}_{2 n}\right)$ in $\mathbf{R}^{2 n}$ with the Clifford vector $\underline{X}=\sum_{j=1}^{n}\left(e_{j} x_{j}+\right.$ $e_{n+j} y_{j}$ ) and we denote by $\underline{X} \mid$ the action of the complex structure $J$ on $\underline{X}$, i.e.

$$
\underline{X} \mid=J[\underline{X}]=\sum_{j=1}^{n}\left(e_{j} y_{j}-e_{n+j} x_{j}\right) .
$$

Note that the vectors $\underline{X}$ and $\underline{X} \mid$ are orthogonal, the Clifford vectors $\underline{X}$ and $\underline{X} \mid$ anticommute. The actions of the projection operators on the Clifford vector $\underline{X}$ then produce the Hermitian Clifford variables $\underline{Z}$ and its Hermitian conjugate $\underline{Z}^{\dagger}$ :

$$
\begin{aligned}
& \underline{Z}=\frac{1}{2}(\mathbf{1}+i J)[\underline{X}]=\frac{1}{2}(\underline{X}+i \underline{X} \mid), \\
& \underline{Z}^{\dagger}=-\frac{1}{2}(\mathbf{1}-i J)[\underline{X}]=-\frac{1}{2}(\underline{X}-i \underline{X} \mid),
\end{aligned}
$$

which can be rewritten in terms of the Witt basis elements as

$$
\underline{Z}=\sum_{j=1}^{n} f_{j} z_{j} \quad \text { and } \quad \underline{Z}^{\dagger}=(\underline{Z})^{\dagger}=\sum_{j=1}^{n} f_{j}^{\dagger} z_{j}^{c},
$$

where $n$ complex variables $z_{j}=x_{j}+i y_{j}$ have been introduced, with complex conjugates $z_{j}^{c}=x_{j}-i y_{j}, j=1, \ldots, n$. Finally, the Hermitian Dirac operators $\partial_{\underline{Z}}$ and $\partial_{\underline{Z}}$ are derived from the orthogonal Dirac operator $\partial_{\underline{X}}$ :

$$
\begin{aligned}
& \partial_{\underline{Z}^{\dagger}}=\frac{1}{4}(\mathbf{1}+i J)\left[\partial_{\underline{X}}\right]=\frac{1}{4}\left(\partial_{\underline{X}}+i \partial_{\underline{X}}\right), \\
& \partial_{\underline{Z}}=-\frac{1}{4}(\mathbf{1}-i J)\left[\partial_{\underline{X}}\right]=-\frac{1}{4}\left(\partial_{\underline{X}}-i \partial_{\underline{X}}\right),
\end{aligned}
$$

where we have introduced

$$
\partial_{\underline{X} \mid}=J\left[\partial_{\underline{X}}\right]=\sum_{j=1}^{n}\left(e_{j} \partial_{y_{j}}-e_{n+j} \partial_{x_{j}}\right) .
$$


In view of the Witt basis, the Hermitian Dirac operators are expressed as

$$
\partial_{\underline{Z}}=\sum_{j=1}^{n} f_{j}^{\dagger} \partial_{\underline{Z}_{j}} \quad \text { and } \quad \partial_{\underline{Z}^{\dagger}}=\left(\partial_{\underline{Z}}\right)^{\dagger}=\sum_{j=1}^{n} f_{j} \partial_{z_{j}^{c}}
$$

involving the classical Cauchy-Riemann operators $\partial_{z_{j}}=\frac{1}{2}\left(\partial_{x_{j}}-i \partial_{y_{j}}\right)$ and their complex conjugates $\partial_{z_{j}^{c}}=\frac{1}{2}\left(\partial_{x_{j}}+i \partial_{y_{j}}\right)$ in the complex $z_{j}$-planes, $j=1, \ldots, n$.

Finally observe that the Hermitian vector variables and Dirac operators are isotropic, since the Witt basis elements are, i.e.

$$
(\underline{Z})^{2}=\left(\underline{Z}^{\dagger}\right)^{2}=0 \quad \text { and } \quad\left(\partial_{\underline{Z}}\right)^{2}=\left(\partial_{\underline{Z}^{\dagger}}\right)^{2}=0
$$

whence the Laplacian $\Delta_{2 n}=-\partial_{X}^{2}=-\partial_{X \mid}^{2}$ allows for the decomposition

$$
\Delta_{2 n}=4\left(\partial_{\underline{Z}} \partial_{\underline{Z}^{\dagger}}+\partial_{\underline{Z}^{\dagger}} \partial_{\underline{Z}}\right)
$$

while also

$$
\underline{Z Z}^{\dagger}+\underline{Z}^{\dagger} \underline{Z}=|\underline{Z}|^{2}=\left|\underline{Z}^{\dagger}\right|^{2}=|\underline{X}|=\left.|\underline{X}|\right|^{2}
$$

For further use, we introduce the Hermitian oriented surface elements $d \sigma_{\underline{Z}}$ and $d \sigma_{\underline{Z}^{\dagger}}$ as follows:

$$
\begin{aligned}
& d \sigma_{\underline{Z}}=-\frac{1}{4}(-1)^{\frac{n(n+1)}{2}}(2 i)^{n}\left(\widetilde{d \sigma}_{\underline{X}}-i \widetilde{d \sigma}_{\underline{X}}\right), \\
& d \sigma_{\underline{Z}^{\dagger}}=-\frac{1}{4}(-1)^{\frac{n(n+1)}{2}}(2 i)^{n}\left(\widetilde{d \sigma}_{\underline{X}}+i \widetilde{d \sigma}_{\underline{X}}\right),
\end{aligned}
$$

where $\widetilde{d \sigma}_{\underline{X}}$ denotes the vector-valued oriented surface element and $\widetilde{d \sigma}_{\underline{X} \mid}=J\left[\widetilde{d \sigma_{\underline{X}}}\right]$. They are explicitly given by means of the following differential forms of order $2 n-1$ :

$$
\begin{aligned}
& \widetilde{d \sigma}_{\underline{X}}=\sum_{j=1}^{n} e_{j}(-1)^{j-1} \widetilde{\widetilde{d x}_{j}}+\sum_{j=1}^{n} e_{n+j}(-1)^{n+j-1} \widetilde{\widetilde{d y_{j}}}, \\
& \widetilde{d \sigma}_{\underline{X} \mid}=\sum_{j=1}^{n} e_{j}(-1)^{n+j-1} \widetilde{\widehat{d y}_{j}}-\sum_{j=1}^{n} e_{n+j}(-1)^{j-1} \widetilde{\widetilde{d x_{j}}},
\end{aligned}
$$

here

$$
\begin{aligned}
& \widetilde{\widetilde{d x}_{j}}=d x_{1} \wedge \cdots \wedge d x_{j-1} \wedge d x_{j+1} \wedge \cdots \wedge d x_{n} \wedge d y_{1} \wedge \cdots \wedge d y_{n}, \\
& \widetilde{\widetilde{d y}_{j}}=d x_{1} \wedge \cdots \wedge d x_{n} \wedge d y_{1} \wedge \cdots \wedge d y_{j-1} \wedge d y_{j+1} \wedge \cdots \wedge d y_{n},
\end{aligned}
$$

and the corresponding oriented volume elements then read

$$
\begin{aligned}
& \widetilde{d V}(\underline{X})=d x_{1} \wedge \cdots \wedge d x_{n} \wedge d y_{1} \wedge \cdots \wedge d y_{n}, \\
& \widetilde{d V}(\underline{X} \mid)=d y_{1} \wedge \cdots \wedge d y_{n} \wedge\left(-d x_{1}\right) \wedge \cdots \wedge\left(-d x_{n}\right) .
\end{aligned}
$$


We also consider the associated volume element $d W\left(\underline{Z}, \underline{Z}^{\dagger}\right)$, defined as

$$
d W\left(\underline{Z}, \underline{Z}^{\dagger}\right)=\left(d z_{1} \wedge d z_{1}^{c}\right) \wedge\left(d z_{2} \wedge d z_{2}^{c}\right) \wedge \cdots \wedge\left(d z_{n} \wedge d z_{n}^{c}\right)
$$

reflecting integration over the respective complex $z_{j}$-planes, $j=1, \ldots, n$. One has

$$
\widetilde{d V}(\underline{X})=(-1)^{\frac{n(n-1)}{2}}\left(\frac{i}{2}\right)^{n} d W\left(\underline{Z}, \underline{Z}^{\dagger}\right) .
$$

We still introduce the matrix

$$
d \Sigma_{\left(\underline{Z}, \underline{Z}^{\dagger}\right)}=\left(\begin{array}{cc}
d \sigma_{\underline{Z}} & -d \sigma_{\underline{Z}^{\dagger}} \\
-d \sigma_{\underline{Z}^{\dagger}} & d \sigma_{\underline{Z}}
\end{array}\right),
$$

which will play the role of the differential form.

Definition 2.1 A continuously differentiable function $f$ on an open region $\Omega$ of $\mathbf{R}^{2 n}$ with values in $\mathbb{C}_{2 n}$ is called a (left) $h$-monogenic function in $\Omega$, iff it satisfies in $\Omega$ the system

$$
\partial_{\underline{X}} f=0=\partial_{\underline{X}} f
$$

or, equivalently, the system

$$
\partial_{\underline{Z}} f=0=\partial_{\underline{Z}} f .
$$

The respective fundamental solutions of $\partial_{\underline{X}}$ and $\partial_{\underline{X} \mid}$ are given by

$$
E(\underline{X})=\frac{1}{\omega_{2 n}} \frac{\bar{X}}{|\underline{X}|^{2 n}}, \quad E \mid(\underline{X})=\frac{1}{\omega_{2 n}} \frac{\bar{X} \mid}{|\underline{X}|^{2 n}}, \quad \underline{X} \in \mathbf{R}^{2 n} \backslash\{0\},
$$

where $\omega_{2 n}$ denotes the area of the unit sphere $S^{2 n-1}$ in $\mathbf{R}^{2 n}$. The transition from Hermitian Clifford analysis to a circulant matrix approach is essentially based on the following observation. Introducing the particular circulant $(2 \times 2)$ matrices

$$
\mathcal{D}_{\left(\underline{Z}, \underline{Z}^{\dagger}\right)}=\left(\begin{array}{cc}
\partial_{\underline{Z}} & \partial_{\underline{Z}^{\dagger}} \\
\partial_{\underline{Z}}^{\dagger} & \partial_{\underline{Z}}
\end{array}\right) \text { and } \quad \mathbf{E}=\left(\begin{array}{cc}
\mathcal{E} & \mathcal{E}^{\dagger} \\
\mathcal{E}^{\dagger} & \mathcal{E}
\end{array}\right) \text {, }
$$

where $\mathcal{E}=-(E+i E \mid)$ and $\mathcal{E}^{\dagger}=(E-i E \mid)$. Then $\mathcal{D}_{\left(\underline{Z}, \underline{Z}^{\dagger}\right)} \mathbf{E}=\delta_{2}^{1}$, where $\delta$ is the diagonal matrix with the Dirac delta distribution $\delta$ on the diagonal, may be considered as a fundamental solution of the matrix operator $\mathcal{D}_{\left(\underline{Z}, \underline{Z}^{\dagger}\right)}$. This has also led to a theory of $\mathbf{H}$-monogenic $(2 \times 2)$ circulant matrix functions, the framework for this theory being as follows. Let $g_{1}, g_{2}$ be continuously differentiable functions defined in $\Omega$ and taking values in $\mathbb{C}_{2 n}$, and consider the corresponding $(2 \times 2)$ circulant matrix function

$$
\mathbf{G}_{2}^{1}(\underline{X})=\left(\begin{array}{ll}
g_{1}(\underline{X}) & g_{2}(\underline{X}) \\
g_{2}(\underline{X}) & g_{1}(\underline{X})
\end{array}\right) .
$$

The ring of such matrix functions over $\mathbb{C}_{2 n}$ is denoted by $\mathbf{C}^{\mathbf{1}} \mathbf{M}^{2 \times 2}$. In what follows, $\mathbf{O}$ will be denoting the matrix in $\mathbf{C}^{\mathbf{1}} \mathbf{M}^{2 \times 2}$ with zero entries. 
Definition 2.2 The matrix function $\mathbf{G}_{2}^{1} \in \mathbf{C}^{\mathbf{1}} \mathbf{M}^{2 \times 2}$ is called (left) $\mathbf{H}$-monogenic in $\Omega$ if and only if it satisfies in $\Omega$ the system $\mathcal{D}_{\left(\underline{Z}, \underline{Z}^{\dagger}\right)}\left[\mathbf{G}_{2}^{1}\right]=\mathbf{O}$.

The notions of continuity, differentiability, and integrability of $\mathbf{G}_{2}^{1} \in \mathbf{C}^{\mathbf{1}} \mathbf{M}^{2 \times 2}$ have the usual component-wise meaning. In particular, we will need to defined in this way the classes $\mathbf{C}^{\mathbf{r}}(\Omega), \mathbf{r} \in \mathbf{N} \backslash\{0\}$, of $\mathbf{r}$ times continuously differentiable functions over some suitable subset $\Omega$ of $\mathbf{R}^{2 n}, \mathbf{C}^{0, \alpha}(\Omega)$ stands for Hölder continuous circulant matrix functions over $\Omega$. We introduce the non-negative function

$$
\left\|\mathbf{G}_{2}^{1}(\underline{X})\right\|=\left(\sum_{i=1}^{2}\left|g_{i}(x)\right|^{2}\right)^{\frac{1}{2}},
$$

where $|\cdot|$ denotes the Clifford norm.

Definition 2.3 The matrix function $\mathbf{G}_{2}^{1} \in \mathbf{C}^{\mathbf{r}} \mathbf{M}^{2 \times 2}(\mathbf{r} \geq 2)$ is called (left) $\mathbf{H}$-2-monogenic in $\Omega$ if and only if it satisfies in $\Omega$ the system $\left(\mathcal{D}_{\left(\underline{Z}, \underline{Z}^{\dagger}\right)}\right)^{2}\left[\mathbf{G}_{2}^{1}\right]=\mathbf{O}$.

In what follows we suppose

$$
\begin{aligned}
& B^{+}(\underline{Y}, R)=\left\{\underline{X} \in \mathbf{R}^{2 n}:|\underline{X}-\underline{Y}|<R\right\}, \\
& B^{-}(\underline{Y}, R)=\left\{\underline{X} \in \mathbf{R}^{2 n}:|\underline{X}-\underline{Y}|>R\right\}, \\
& \partial B(\underline{Y}, R)=\left\{\underline{X} \in \mathbf{R}^{2 n}:|\underline{X}-\underline{Y}|=R\right\},
\end{aligned}
$$

with $R>0$.

\section{Some properties for $\mathrm{H}$-monogenic circulant $(2 \times 2)$ matrix functions}

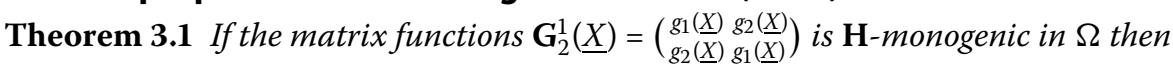

$$
\frac{2 n}{\omega_{2 n} R^{2 n}} \int_{B(\underline{Y}, R)} \mathbf{G}_{2}^{1}(\underline{X}) \widetilde{d V}(\underline{X})=\mathbf{G}_{2}^{1}(\underline{Y})
$$

for each $R>0$ such that $\bar{B}(\underline{Y}, R) \subset \Omega$.

Proof Take $R>0$ such that $\bar{B}(\underline{Y}, R) \subset \Omega$. Apply Hermitian Cauchy's integral formula I (in [17]). On the ball $\bar{B}(\underline{Y}, R)$, we have

$$
\begin{aligned}
\mathbf{G}_{2}^{1}(\underline{Y}) & =(-1)^{\frac{n(n+1)}{2}}\left(-\frac{i}{2}\right)^{n} \int_{\partial B(\underline{Y}, R)} \mathbf{E}(\underline{Z}-\underline{V}) d \Sigma_{\left(\underline{Z}, \underline{Z}^{\dagger}\right)} \mathbf{G}_{2}^{1}(\underline{X}) \\
& =\frac{2}{\omega_{2 n} R^{2 n}}(-1)^{\frac{n(n+1)}{2}}\left(-\frac{i}{2}\right)^{n} \int_{\partial B(\underline{Y}, R)} \mathbf{G}_{\underline{Z}-\underline{V}} d \Sigma_{\left(\underline{Z}, \underline{Z}^{\dagger}\right)} \mathbf{G}_{2}^{1}(\underline{X}),
\end{aligned}
$$

where

$$
\mathbf{G}_{\underline{Z}-\underline{V}}=\left(\begin{array}{cc}
\underline{Z}-\underline{V} & \underline{Z}^{\dagger}-\underline{V}^{\dagger} \\
\underline{Z}^{\dagger}-\underline{V}^{\dagger} & \underline{Z}-\underline{V}
\end{array}\right) .
$$


As $\left[\mathbf{G}_{\underline{Z}-\underline{V}}\right] \mathcal{D}_{\left(\underline{Z}, \underline{Z}^{\dagger}\right)}=\left(\begin{array}{ll}n & 0 \\ 0 & n\end{array}\right)$, we apply the Hermitian Clifford-Stokes theorem (in [17]),

$$
\mathbf{G}_{2}^{1}(\underline{Y})=\frac{2}{\omega_{2 n} R^{2 n}} \int_{B(\underline{Y}, R)}\left(\begin{array}{ll}
n & 0 \\
0 & n
\end{array}\right) \mathbf{G}_{2}^{1}(\underline{X}) \widetilde{d V}(\underline{X}) .
$$

The result follows.

The notions of continuity, differentiability, and integrability of $\mathbf{G}_{2}^{1}(\underline{X})$ have the usual component-wise meaning.

Theorem 3.2 (Liouville theorem) If the matrix function $\mathbf{G}_{2}^{1} \in \mathbf{C}^{\mathbf{1}} \mathbf{M}^{2 \times 2}$ is $\mathbf{H}$-monogenic in $\mathbf{R}^{2 n}$ and satisfies $\| \mathbf{G}_{2}^{1}\left(\underline{X)} \| \leq M\right.$ for all $\underline{X} \in \mathbf{R}^{2 n}$ then $\mathbf{G}_{2}^{1}(\underline{X)}$ must be a constant circulant matrix in $\mathbf{R}^{2 n}$.

Proof By Theorem 3.1, we have

$$
\begin{aligned}
\left\|\mathbf{G}_{2}^{1}(\underline{Y})-\mathbf{G}_{2}^{1}(0)\right\| & =\frac{2 n}{\omega_{2 n} R^{2 n}}\left\|\int_{B(\underline{Y}, R)} \mathbf{G}_{2}^{1}(\underline{X}) \widetilde{d V}(\underline{X})-\int_{B(0, R)} \mathbf{G}_{2}^{1}(\underline{X}) \widetilde{d V}(\underline{X})\right\| \\
& \leq M \frac{V\left(D_{R}\right)}{V(B(0, R))},
\end{aligned}
$$

where $D_{R}$ denotes the symmetric difference of $B(\underline{Y}, R)$ and $B(0, R), V(\cdot)$ is Lebesgue volume measure on $\mathbf{R}^{2 n}$, so that $D_{R}=[B(\underline{Y}, R) \cup B(0, R)] \backslash[B(\underline{Y}, R) \cap B(0, R)]$. The last expression above tends to 0 as $R \rightarrow \infty$. Thus $\mathbf{G}_{2}^{1}\left(\underline{Y)}=\mathbf{G}_{2}^{1}(0)\right.$ and so $\mathbf{G}_{2}^{1}(\underline{Y})$ is a constant circulant matrix.

Theorem 3.3 (Maximum modulus theorem) Let the matrix functions $\mathbf{G}_{2}^{1}(\underline{X})$ be a $\mathbf{H}$-monogenic in the open and connected set $\Omega$. If there exists a point $\underline{A} \in \Omega$ such that

$$
\| \mathbf{G}_{2}^{1}\left(\underline{X)}\|\leq\| \mathbf{G}_{2}^{1}(\underline{A}) \|\right.
$$

for all $\underline{X} \in \Omega$, then $\mathbf{G}_{2}^{1}$ must be constant circulant matrix in $\Omega$.

Proof Put $\left\|\mathbf{G}_{2}^{1}(\underline{A})\right\|=\lambda$ and consider the subset $\Omega_{\lambda}$ of $\Omega$ given by

$$
\Omega_{\lambda}=\left\{\underline{X} \in \Omega \mid\left\|\mathbf{G}_{2}^{1}(\underline{X})\right\|=\lambda\right\} .
$$

Since $\underline{A} \in \Omega_{\lambda}$, then $\Omega_{\lambda} \neq \varnothing$. So let $\underline{Y} \in \Omega \backslash \Omega_{\lambda}$; this implies that $\left\|\mathbf{G}_{2}^{1}(\underline{Y})\right\|<\lambda$. As $\left\|\mathbf{G}_{2}^{1}(\underline{X})\right\|$ is continuous in $\Omega$, there exists an $R^{\prime}>0$ such that $B\left(\underline{Y}, R^{\prime}\right) \subset \Omega \backslash \Omega_{\lambda}$. This means that $\Omega_{\lambda}$ is relatively closed in $\Omega$.

Now take $\underline{Y}^{\prime} \in \Omega_{\lambda}$ and $R>0$ such that $B\left(\underline{Y}^{\prime}, R\right) \subset \Omega$. By Theorem 3.1, we have

$$
\mathbf{G}_{2}^{1}\left(\underline{Y^{\prime}}\right)=\frac{2 n}{\omega_{2 n} R^{2 n}} \int_{B\left(\underline{Y^{\prime}}, R\right)} \mathbf{G}_{2}^{1}(\underline{X}) \widetilde{d V}(\underline{X})
$$

i.e.

$$
\left(\begin{array}{ll}
g_{1}\left(\underline{Y}^{\prime}\right) & g_{2}\left(\underline{Y}^{\prime}\right) \\
g_{2}\left(\underline{Y}^{\prime}\right) & g_{1}\left(\underline{Y}^{\prime}\right)
\end{array}\right)=\frac{2 n}{\omega_{2 n} R^{2 n}}\left(\begin{array}{ll}
\int_{B\left(\underline{Y}^{\prime}, R\right)} g_{1}(\underline{X}) \widetilde{d V}(\underline{X}) & \int_{B\left(\underline{Y}^{\prime}, R\right)} g_{2}(\underline{X}) \widetilde{d V}(\underline{X)} \\
\int_{B\left(\underline{Y}^{\prime}, R\right)} g_{2}(\underline{X}) \widetilde{d V}(\underline{X}) & \int_{B\left(\underline{(}^{\prime}, R\right)} g_{1}(\underline{X}) \widetilde{d V}(\underline{X})
\end{array}\right),
$$


we then have

$$
\lambda^{2}=\left\|\mathbf{G}_{2}^{1}\left(\underline{Y}^{\prime}\right)\right\|^{2}=2^{2 n}\left(\frac{2 n}{\omega_{2 n} R^{2 n}}\right)^{2} \sum_{i=1}^{2} \sum_{A}\left|\int_{B\left(\underline{\left.Y^{\prime}, R\right)}\right.} g_{i_{A}}(\underline{X}) \widetilde{d V}(\underline{X})\right|^{2}
$$

Applying Hölder's inequality,

$$
\begin{aligned}
\lambda^{2} & \leq 2^{2 n} \frac{4 n^{2}}{\omega_{2 n}^{2} R^{4 n}} \sum_{i=1}^{2} \sum_{A}\left(\int_{B\left(\underline{Y^{\prime}}, R\right)} \widetilde{d V}(\underline{X})\right)\left(\int_{B\left(\underline{Y^{\prime}}, R\right)}\left|g_{i_{A}}(\underline{X})\right|^{2} \widetilde{d V}(\underline{X})\right) \\
& \leq \frac{2 n}{\omega_{2 n} R^{2 n}} \sum_{i=1}^{2} \int_{B\left(\underline{Y}^{\prime}, R\right)}\left|g_{i}(\underline{X})\right|^{2} \widetilde{d V}(\underline{X}) \\
& =\frac{2 n}{\omega_{2 n} R^{2 n}} \int_{B\left(\underline{\underline{Y}^{\prime}}, R\right)}\left\|\mathbf{G}_{2}^{1}(\underline{X})\right\|^{2} \widetilde{d V}(\underline{X}) .
\end{aligned}
$$

Hence

$$
0 \leq \frac{2 n}{\omega_{2 n} R^{2 n}} \int_{B\left(\underline{Y}^{\prime}, R\right)}\left(\left\|\mathbf{G}_{2}^{1}(\underline{X})\right\|-\lambda^{2}\right) \widetilde{d V}(\underline{X}) \leq 0
$$

which yields $\left\|\mathbf{G}_{2}^{1}\right\|=\lambda$ for all $\underline{X} \in \stackrel{o}{B}\left(\underline{Y}^{\prime}, R\right)$, this means that $\stackrel{o}{B}\left(\underline{Y}^{\prime}, R\right) \subset \Omega_{\lambda}$ and hence that $\Omega_{\lambda}$ is relatively open in $\Omega$. As $\Omega$ is supposed to be connected it follows that $\Omega=\Omega_{\lambda}$.

Now if $\lambda=0$ then clearly $\mathbf{G}_{2}^{1}(\underline{X})=\mathbf{O}$ for all $\underline{X} \in \Omega$. For $\lambda \neq 0$, since $\mathbf{G}_{2}^{1}(\underline{X})$ is $\mathbf{H}$-monogenic in $\Omega$, we have

$$
\begin{aligned}
\mathbf{O} & =4\left(\mathcal{D}_{\left(\underline{Z}, \underline{Z}^{\dagger}\right)}\right)^{\dagger}\left(\mathcal{D}_{\left(\underline{Z}, \underline{Z}^{\dagger}\right)}\left(\begin{array}{ll}
g_{1}(\underline{X}) & g_{2}(\underline{X}) \\
g_{2}(\underline{X}) & g_{1}(\underline{X})
\end{array}\right)\right. \\
& =\left(\begin{array}{cc}
\Delta_{2 n} & 0 \\
0 & \Delta_{2 n}
\end{array}\right)\left(\begin{array}{ll}
g_{1}(\underline{X}) & g_{2}(\underline{X}) \\
g_{2}(\underline{X}) & g_{1}(\underline{X})
\end{array}\right),
\end{aligned}
$$

then for all $\underline{X} \in \Omega, \Delta_{2 n} g_{1}(\underline{X})=0$ and $\Delta_{2 n} g_{2}(\underline{X})=0$. Hence we obtain $\Delta_{2 n} g_{1_{A}}(\underline{X})=0$ and $\Delta_{2 n} g_{2_{A}}(\underline{X})=0$ for all $\underline{X} \in \Omega$. For all $\underline{X} \in \Omega$ we have

$$
2^{2 n} \sum_{i=1}^{2} \sum_{A}\left|g_{i_{A}}(\underline{X})\right|^{2}=\lambda^{2}
$$

i.e.

$$
2^{2 n} \sum_{i=1}^{2} \sum_{A} g_{1_{A}}(\underline{X}) \overline{g_{i_{A}}(\underline{X})}=\lambda^{2}
$$

and by (3.7), differentiating twice, we get

$$
\sum_{i=1}^{2} \sum_{A} \partial_{x_{j}^{2}}^{2} g_{i_{A}}(\underline{X}) \overline{g_{i_{A}}(\underline{X})}+\sum_{i=1}^{2} \sum_{A} g_{i_{A}}\left(\underline{X)} \overline{\partial_{x_{j}^{2}}^{2} g_{i_{A}}(\underline{X})}+2 \sum_{i=1}^{2} \sum_{A}\left|\partial_{x_{j}} g_{i_{A}}(\underline{X})\right|^{2}=0 .\right.
$$


Summing up over $j=1,2, \ldots, 2 n$ yields

$$
\begin{aligned}
& \sum_{i=1}^{2} \sum_{A}\left(\Delta_{2 n} g_{i_{A}}(\underline{X})\right) \overline{\left(g_{i_{A}}(\underline{X})\right)}+\sum_{i=1}^{2} \sum_{A} g_{i_{A}}(\underline{X}) \overline{\left(\Delta_{2 n} g_{i_{A}}(\underline{X})\right)} \\
& \quad+2 \sum_{i=1}^{2} \sum_{j, A}\left|\partial_{x_{j}} g_{i_{A}}(\underline{X})\right|^{2}=0,
\end{aligned}
$$

we have $\partial_{x_{j}} g_{i_{A}}(\underline{X})=0(i=1,2)$ in $\Omega$ for all $j=1,2, \ldots, 2 n$ all $A \in \mathcal{P} N$. Thus $g_{1}(\underline{X}), g_{2}(\underline{X})$ are constants in $\Omega$. The result follows.

Corollary 3.4 Let $\Omega$ be a bounded open set in $\mathbf{R}^{2 n}$ and suppose that $g_{1}(\underline{X}), g_{2}(\underline{X})$ are func-

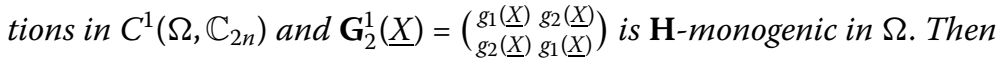

$$
\sup _{\underline{X} \in \bar{\Omega}}\left\|\mathbf{G}_{2}^{1}(\underline{X})\right\|=\sup _{\underline{X} \in \partial \Omega}\left\|\mathbf{G}_{2}^{1}(\underline{X})\right\|
$$

\section{Higher order Hermitian Borel-Pompeiu formula in Hermitian Clifford analysis}

Integral representation formulas in Clifford analysis have been well developed in [3, 2325], etc. These integral representation formulas are powerful tools. In this section, we get the explicit expression of the kernel function for $\left(\mathcal{D}_{\left(\underline{Z}, \underline{Z}^{\dagger}\right)}\right)^{2}$ and then get the explicit integral representation formulas for functions in Hermitian Clifford analysis. These explicit integral representation formulas play an important role in studying the further properties of the functions in Hermitian Clifford analysis.

In what follows, we denote

$$
\begin{aligned}
& \mathbf{I}=\left(\begin{array}{ll}
1 & 0 \\
0 & 1
\end{array}\right), \\
& \widetilde{\mathbf{I}}=\left(\begin{array}{ll}
0 & 1 \\
1 & 0
\end{array}\right), \\
& \mathbf{G}_{\underline{Z}-\underline{V}}=\left(\begin{array}{cc}
\underline{Z}-\underline{V} & \underline{Z}^{\dagger}-\underline{V^{\dagger}} \\
\underline{Z}-\underline{V^{\dagger}} & \underline{Z}-\underline{V}
\end{array}\right), \\
& \mathbf{G}_{(\underline{X}, \underline{Y})}=\left(\begin{array}{cc}
0 & |\underline{X}-\underline{Y}|^{2} \\
|\underline{X}-\underline{Y}|^{2} & 0
\end{array}\right), \\
& \mathbf{E}_{1}(\underline{Z}-\underline{V})=\frac{1}{\omega_{2 n}(2-2 n)}\left(\begin{array}{cc}
0 & \frac{4}{|\underline{X}-\underline{Y}|^{2 n-2}} \\
\frac{1}{|\underline{Y}|^{2 n-2}} & 0
\end{array}\right), \quad \underline{X} \in \mathbf{R}^{2 n} \backslash\{\underline{Y}\},
\end{aligned}
$$

where $\omega_{2 n}$ denotes the area of the unit sphere in $\mathbf{R}^{2 n}$.

Lemma 4.1 Let $\mathbf{E}_{1}(\underline{Z}-\underline{V})$ be as in (4.5). Then $\left[\mathbf{E}_{1}(\underline{Z}-\underline{V})\right] \mathcal{D}_{\left(\underline{Z}, \underline{Z^{\dagger}}\right)}=\mathbf{E}(\underline{Z}-\underline{V})$.

Proof The identity is obtained by straightforward calculation.

Lemma 4.2 Denote $\partial_{\underline{X}}=\sum_{j=1}^{n}\left(e_{j} \partial_{x_{j}}+e_{n+j} \partial_{y_{j}}\right)$, $\partial_{\underline{X} \mid}=\sum_{j=1}^{n}\left(e_{j} \partial_{y_{j}}-e_{n+j} \partial_{x_{j}}\right)$, then 
1.

$$
\partial_{\underline{X}}\left(|\underline{X}-\underline{Y}|^{2}\right)=2(\underline{X}-\underline{Y}),
$$

2.

$$
\partial_{\underline{X} \mid}\left(|\underline{X}-\underline{Y}|^{2}\right)=2(\underline{X}|-\underline{Y}|) .
$$

Lemma 4.3 Let $\mathbf{G}_{(\underline{X}, \underline{Y})}$ and $\mathbf{G}_{\underline{\underline{Z}}-\underline{\underline{V}}}$ be as in (4.4) and (4.3). Then

$$
\mathbf{G}_{(\underline{X}, \underline{Y})} \mathcal{D}_{\left(\underline{Z}, \underline{Z}^{\dagger}\right)}=\mathbf{G}_{\underline{Z}-\underline{V}}
$$

Proof In view of Lemma 4.2, the identity is obtained by straightforward calculation.

Theorem 4.4 (Higher order Hermitian Borel-Pompeiu formula) Suppose $\Gamma \subset \Omega$ is a $2 n$-dimensional compact differentiable and oriented manifold with $C^{\infty}$ smooth boundary $\partial \Gamma, g_{1}$ and $g_{2}$ are functions in $C^{2}\left(\Omega, \mathbb{C}_{2 n}\right)$ and $\mathbf{G}_{2}^{1}(\underline{X})=\left(\begin{array}{l}g_{1}(\underline{X}) \\ g_{2}(\underline{X}) \\ g_{1}(\underline{X})\end{array}\right)$ is the matrix function. It then follows that

$$
\begin{aligned}
\int_{\partial \Gamma} \mathbf{E}(\underline{Z}-\underline{V}) d \Sigma_{\left(\underline{Z}, \underline{Z}^{\dagger}\right)} \mathbf{G}_{2}^{1}(\underline{X})-\int_{\partial \Gamma} \mathbf{E}_{1}(\underline{Z}-\underline{V}) d \Sigma_{\left(\underline{Z}, \underline{Z}^{\dagger}\right)} \mathcal{D}_{\left(\underline{Z}, \underline{Z}^{\dagger}\right)} \mathbf{G}_{2}^{1}(\underline{X}) \\
+\int_{\Gamma} \mathbf{E}_{1}(\underline{Z}-\underline{V})\left[\left(\mathcal{D}_{\left(\underline{Z}, \underline{Z}^{\dagger}\right)}\right)^{2} \mathbf{G}_{2}^{1}(\underline{X})\right] d W_{\left(\underline{Z}, \underline{Z}^{\dagger}\right)} \\
= \begin{cases}\mathbf{O}, & \text { if } \underline{Y} \in \Gamma^{-}, \\
(-1)^{\frac{n(n+1)}{2}}(2 i)^{n} \mathbf{G}_{2}^{1}(\underline{Y}), & \text { if } \underline{Y} \in \Gamma^{+} .\end{cases}
\end{aligned}
$$

Proof First let $\underline{Y}=\underline{V}-\underline{V}^{\dagger} \in \Gamma^{-}$. It then follows from the Stokes formula, which can be found in [17], that we have

$$
\begin{aligned}
\int_{\Gamma} & \mathbf{E}_{1}(\underline{Z}-\underline{V})\left[\left(\mathcal{D}_{\left(\underline{Z}, \underline{Z}^{\dagger}\right)}\right)^{2} \mathbf{G}_{2}^{1}(\underline{X})\right] d W_{\left(\underline{Z}, \underline{Z}^{\dagger}\right)} \\
= & \int_{\partial \Gamma} \mathbf{E}_{1}(\underline{Z}-\underline{V}) d \Sigma_{\left(\underline{Z}, \underline{Z}^{\dagger}\right)} \mathcal{D}_{\left(\underline{Z}, \underline{Z}^{\dagger}\right)} \mathbf{G}_{2}^{1}(\underline{X}) \\
& -\int_{\Gamma}\left[\mathbf{E}_{1}(\underline{Z}-\underline{V}) \mathcal{D}_{\left(\underline{Z}, \underline{Z}^{\dagger}\right)}\right]\left[\mathcal{D}_{\left(\underline{Z}, \underline{Z}^{\dagger}\right)} \mathbf{G}_{2}^{1}(\underline{X})\right] d W_{\left(\underline{Z}, \underline{Z}^{\dagger}\right)} \\
= & \int_{\partial \Gamma} \mathbf{E}_{1}(\underline{Z}-\underline{V}) d \Sigma_{\left(\underline{Z}, \underline{Z}^{\dagger}\right)} \mathcal{D}_{\left(\underline{Z}, \underline{Z}^{\dagger}\right)} \mathbf{G}_{2}^{1}(\underline{X})-\int_{\Gamma} \mathbf{E}(\underline{Z}-\underline{V})\left[\mathcal{D}_{\left(\underline{Z}, \underline{Z}^{\dagger}\right)} \mathbf{G}_{2}^{1}(\underline{X})\right] d W_{\left(\underline{Z}, \underline{Z}^{\dagger}\right)} \\
= & \int_{\partial \Gamma} \mathbf{E}_{1}(\underline{Z}-\underline{V}) d \Sigma_{\left(\underline{Z}, \underline{Z}^{\dagger}\right)} \mathcal{D}_{\left(\underline{Z}, \underline{Z}^{\dagger}\right)} \mathbf{G}_{2}^{1}(\underline{X})-\int_{\partial \Gamma} \mathbf{E}(\underline{Z}-\underline{V}) d \Sigma_{\left(\underline{Z}, \underline{Z}^{\dagger}\right)} \mathbf{G}_{2}^{1}(\underline{X}),
\end{aligned}
$$

then the left-hand side of the stated formula apparently equals zero.

Now, let $\underline{Y}=\underline{V}-\underline{V}^{\dagger} \in \Gamma^{+}$and take $R>0$ such that $B(\underline{Y}, R) \subset \Gamma^{+}$. Invoking the previous case, we may then write

$$
\begin{aligned}
& \int_{\partial(\Gamma \backslash B(\underline{Y}, R))} \mathbf{E}(\underline{Z}-\underline{V}) d \Sigma_{\left(\underline{Z}, \underline{Z}^{\dagger}\right)} \mathbf{G}_{2}^{1}(\underline{X})-\int_{\partial(\Gamma \backslash B(\underline{Y}, R))} \mathbf{E}_{1}(\underline{Z}-\underline{V}) d \Sigma_{\left(\underline{Z}, \underline{Z}^{\dagger}\right)} \mathcal{D}_{\left(\underline{Z}, \underline{Z}^{\dagger}\right)} \mathbf{G}_{2}^{1}(\underline{X}) \\
& \quad+\int_{\Gamma \backslash B(\underline{Y}, R)} \mathbf{E}_{1}(\underline{Z}-\underline{V})\left[\left(\mathcal{D}_{\left(\underline{Z}, \underline{Z}^{\dagger}\right)}\right)^{2} \mathbf{G}_{2}^{1}(\underline{X})\right] d W_{\left(\underline{Z}, \underline{Z}^{\dagger}\right)}=\mathbf{O} .
\end{aligned}
$$


Here we take the limits for $R \rightarrow 0$. In view of the weak singularity of $\frac{1}{\omega_{2 n}(2-2 n)} \frac{4}{|\underline{X}-\underline{Y}|^{2 n-2}}$ the third term of (4.11) yields

$$
\begin{gathered}
\lim _{R \rightarrow 0} \int_{\Gamma \backslash B(\underline{Y}, R)} \mathbf{E}_{1}(\underline{Z}-\underline{V})\left[\left(\mathcal{D}_{\left(\underline{Z}, \underline{Z}^{\dagger}\right)}\right)^{2} \mathbf{G}_{2}^{1}(\underline{X})\right] d W_{\left(\underline{Z}, \underline{Z}^{\dagger}\right)} \\
=\int_{\Gamma} \mathbf{E}_{1}(\underline{Z}-\underline{V})\left[\left(\mathcal{D}_{\left(\underline{Z}, \underline{Z}^{\dagger}\right)}\right)^{2} \mathbf{G}_{2}^{1}(\underline{X})\right] d W_{\left(\underline{Z}, \underline{Z}^{\dagger}\right)}
\end{gathered}
$$

since the integrand only contains functions which are integrable on $\Gamma$. Furthermore we may write

$$
\begin{aligned}
& \int_{\partial(\Gamma \backslash B(\underline{\underline{Y}}, R))} \mathbf{E}(\underline{Z}-\underline{V}) d \Sigma_{\left(\underline{Z}, \underline{Z}^{\dagger}\right)} \mathbf{G}_{2}^{1}(\underline{X}) \\
&-\int_{\partial(\Gamma \backslash B(\underline{\underline{Y}, R))}} \mathbf{E}_{1}(\underline{Z}-\underline{V}) d \Sigma_{\left(\underline{Z}, \underline{Z}^{\dagger}\right)} \mathcal{D}_{\left(\underline{\underline{Z}}, \underline{Z}^{\dagger}\right)} \mathbf{G}_{2}^{1}(\underline{X}) \\
&=\int_{\partial \Gamma} \mathbf{E}(\underline{Z}-\underline{V}) d \Sigma_{\left(\underline{Z}, \underline{Z}^{\dagger}\right)} \mathbf{G}_{2}^{1}(\underline{X})-\int_{\partial \Gamma} \mathbf{E}_{1}(\underline{Z}-\underline{V}) d \Sigma_{\left(\underline{Z}, \underline{Z}^{\dagger}\right)} \mathcal{D}_{\left(\underline{Z}, \underline{Z}^{\dagger}\right)} \mathbf{G}_{2}^{1}(\underline{X}) \\
&- {\left[\int_{\partial B(\underline{Y}, R)} \mathbf{E}(\underline{Z}-\underline{V}) d \Sigma_{\left(\underline{Z}, \underline{Z}^{\dagger}\right)} \mathbf{G}_{2}^{1}(\underline{X})\right.} \\
&\left.-\int_{\partial B(\underline{Y}, R)} \mathbf{E}_{1}(\underline{Z}-\underline{V}) d \Sigma_{\left(\underline{Z}, \underline{Z}^{\dagger}\right)} \mathcal{D}_{\left(\underline{Z}, \underline{Z}^{\dagger}\right)} \mathbf{G}_{2}^{1}(\underline{X})\right],
\end{aligned}
$$

we denote

$$
\begin{aligned}
\Upsilon:= & \int_{\partial B(\underline{Y}, R)} \mathbf{E}(\underline{Z}-\underline{V}) d \Sigma_{\left(\underline{Z}, \underline{Z}^{\dagger}\right)} \mathbf{G}_{2}^{1}(\underline{X}) \\
& -\int_{\partial B(\underline{Y}, R)} \mathbf{E}_{1}(\underline{Z}-\underline{V}) d \Sigma_{\left(\underline{Z}, \underline{Z}^{\dagger}\right)} \mathcal{D}_{\left(\underline{Z}, \underline{Z}^{\dagger}\right)} \mathbf{G}_{2}^{1}(\underline{X}) .
\end{aligned}
$$

Combining the Stokes formula in Hermitian Clifford analysis with

$$
\left(\begin{array}{cc}
\underline{Z}-\underline{V} & \underline{Z}^{\dagger}-\underline{V}^{\dagger} \\
\underline{Z}^{\dagger}-\underline{V}^{\dagger} & \underline{Z}-\underline{V}
\end{array}\right) \mathcal{D}_{\left(\underline{Z}, \underline{Z}^{\dagger}\right)}=\left(\begin{array}{cc}
n & 0 \\
0 & n
\end{array}\right),
$$

we get

$$
\begin{aligned}
\Upsilon= & \frac{2 n}{\omega_{2 n} R^{2 n}} \int_{B(\underline{Y}, R)} \mathbf{G}_{2}^{1}(\underline{X}) d W_{\left(\underline{Z}, \underline{Z}^{\dagger}\right)}+\int_{B(\underline{Y}, R)} \mathbf{G}_{\underline{Z}-\underline{V}}\left[\mathcal{D}_{\left(\underline{Z}, \underline{Z}^{\dagger}\right)} \mathbf{G}_{2}^{1}(\underline{X})\right] d W_{\left(\underline{Z}, \underline{Z}^{\dagger}\right)} \\
& +\frac{4}{(2 n-2) \omega_{2 n} R^{2 n-2}} \int_{B(\underline{Y}, R)} \widetilde{\mathbf{I}}\left[\left(\mathcal{D}_{\left(\underline{Z}, \underline{Z}^{\dagger}\right)}\right)^{2} \mathbf{G}_{2}^{1}(\underline{X})\right] d W_{\left(\underline{Z}, \underline{Z}^{\dagger}\right)},
\end{aligned}
$$

where $\widetilde{\mathbf{I}}$ is defined as in (4.2).

It is clear that

$$
\lim _{R \rightarrow 0} \Upsilon=(-1)^{\frac{n(n+1)}{2}}(2 i)^{n} \mathbf{G}_{2}^{1}(\underline{Y}) .
$$

Then the result follows. 
Theorem 4.5 If the matrix function $\mathbf{G}_{2}^{1}$ is $\mathbf{H}-2$-monogenic in $\Omega$ then

$$
\begin{aligned}
\int_{\partial \Gamma} \mathbf{E}(\underline{Z}-\underline{V}) d \Sigma_{\left(\underline{Z}, \underline{Z}^{\dagger}\right)} \mathbf{G}_{2}^{1}(\underline{X})-\int_{\partial \Gamma} \mathbf{E}_{1}(\underline{Z}-\underline{V}) d \Sigma_{\left(\underline{Z}, \underline{Z}^{\dagger}\right)} \mathcal{D}_{\left(\underline{Z}, \underline{Z}^{\dagger}\right)} \mathbf{G}_{2}^{1}(\underline{X}) \\
\quad= \begin{cases}\mathbf{O}, & \text { if } \underline{Y} \in \Gamma^{-}, \\
(-1)^{\frac{n(n+1)}{2}}(2 i)^{n} \mathbf{G}_{2}^{1}(\underline{Y}), & \text { if } \underline{Y} \in \Gamma^{+} .\end{cases}
\end{aligned}
$$

Proof Since $\mathbf{G}_{2}^{1}$ is $\mathbf{H}$-2-monogenic in $\Omega$, in view of Theorem 4.4, the result follows.

Theorem 4.6 Let $B(\underline{a}, R)$ be an open ball centered at a with radius $R$ in $\mathbf{R}^{2 n}, \mathbf{G}_{2}^{1} \in$ $\mathbf{C}^{2}(B(\underline{a}, R)) \cap \mathbf{C}^{1}(\overline{B(\underline{a}, R)})$ and the matrix function $\mathbf{G}_{2}^{1}$ is $\mathbf{H}$-2-monogenic in $B(\underline{a}, R)$, then for all $\underline{Y} \in B(\underline{a}, R)$

$$
\begin{aligned}
& \int_{\partial B(\underline{a}, R)} \mathbf{E}(\underline{Z}-\underline{V}) d \Sigma_{\left(\underline{Z}, \underline{Z}^{\dagger}\right)} \mathbf{G}_{2}^{1}\left(\underline{X)}-\int_{\partial B(\underline{a}, R)} \mathbf{E}_{1}(\underline{Z}-\underline{V}) d \Sigma_{\left(\underline{Z}, \underline{Z}^{\dagger}\right)} \mathcal{D}_{\left(\underline{Z}, \underline{Z}^{\dagger}\right)} \mathbf{G}_{2}^{1}(\underline{X})\right. \\
& =(-1)^{\frac{n(n+1)}{2}}(2 i)^{n} \mathbf{G}_{2}^{1}(\underline{Y}) .
\end{aligned}
$$

Theorem 4.7 (Mean value theorem for $\mathbf{H}$-2-monogenic matrix function) If the matrix function $\mathbf{G}_{2}^{1}(\underline{X})=\left(\begin{array}{ll}g_{1}(\underline{X}) & g_{2}(\underline{X}) \\ g_{2}(\underline{X}) & g_{1}(\underline{X})\end{array}\right)$ is $\mathbf{H}$-2-monogenic in $\Omega$ then

$$
\frac{2 n}{\omega_{2 n} R^{2 n}} \int_{B(\underline{Y}, R)} \mathbf{G}_{2}^{1}(\underline{X}) \widetilde{d V}(\underline{X})=\mathbf{G}_{2}^{1}(\underline{Y})
$$

for each $R>0$ such that $\bar{B}(\underline{Y}, R) \subset \Omega$.

Proof Take $R>0$ such that $\bar{B}(\underline{Y}, R) \subset \Omega$, by Theorem 4.5 we get

$$
\begin{aligned}
(-1)^{\frac{n(n+1)}{2}}(2 i)^{n} \mathbf{G}_{2}^{1}(\underline{Y})= & \int_{\partial B(\underline{Y}, R)} \mathbf{E}(\underline{Z}-\underline{V}) d \Sigma_{\left(\underline{Z}, \underline{Z}^{\dagger}\right)} \mathbf{G}_{2}^{1}(\underline{X}) \\
& -\int_{\partial B(\underline{Y}, R)} \mathbf{E}_{1}(\underline{Z}-\underline{V}) d \Sigma_{\left(\underline{Z}, \underline{Z}^{\dagger}\right)} \mathcal{D}_{\left(\underline{Z}, \underline{Z}^{\dagger}\right)} \mathbf{G}_{2}^{1}(\underline{X}) \\
= & \frac{2}{\omega_{2 n} R^{2 n}} \int_{\partial B(\underline{Y}, R)} \mathbf{G}_{\underline{Z}-\underline{V}} d \Sigma_{\left(\underline{Z}, \underline{Z}^{\dagger}\right)} \mathbf{G}_{2}^{1}(\underline{X}) \\
& +\frac{4}{(2 n-2) \omega_{2 n} R^{2 n-2}} \int_{\partial B(\underline{Y}, R)} \widetilde{\mathbf{I}} d \Sigma_{\left(\underline{Z}, \underline{Z}^{\dagger}\right)} \mathcal{D}_{\left(\underline{Z}, \underline{Z}^{\dagger}\right)} \mathbf{G}_{2}^{1}(\underline{X}) \\
:= & \Upsilon_{1} .
\end{aligned}
$$

Combining with the Stokes formula in Hermitian Clifford analysis, $\mathbf{G}_{2}^{1}$ is $\mathbf{H}$-2-monogenic in $\Omega$, Lemma 4.3 with $(-2 i)^{n}(-1)^{\frac{n(n-1)}{2}} \widetilde{d V}(\underline{X})=d W\left(\underline{Z}, \underline{Z}^{\dagger}\right)$, we have

$$
\begin{aligned}
\Upsilon_{1}= & \frac{2 n}{\omega_{2 n} R^{2 n}} \int_{B(\underline{Y}, R)} \mathbf{G}_{2}^{1}(\underline{X}) d W\left(\underline{Z}, \underline{Z}^{\dagger}\right) \\
& +\frac{2}{\omega_{2 n} R^{2 n}} \int_{B(\underline{Y}, R)} \mathbf{G}_{\underline{Z}-\underline{V}}\left[\mathcal{D}_{\left(\underline{Z}, \underline{Z}^{\dagger}\right)} \mathbf{G}_{2}^{1}(\underline{X})\right] d W\left(\underline{Z}, \underline{Z}^{\dagger}\right) \\
= & \frac{2 n}{\omega_{2 n} R^{n}}(-2 i)^{2 n}(-1)^{\frac{n(n-1)}{2}} \int_{B(\underline{Y}, R)} \mathbf{G}_{2}^{1}(\underline{X}) \widetilde{d V}(\underline{X})
\end{aligned}
$$




$$
\begin{aligned}
& +\frac{2}{\omega_{2 n} R^{2 n}} \int_{B(\underline{Y}, R)}\left[\mathbf{G}_{(\underline{X}, \underline{Y})} \mathcal{D}_{\left(\underline{Z}, \underline{Z}^{\dagger}\right)}\right]\left[\mathcal{D}_{\left(\underline{Z}, \underline{Z}^{\dagger}\right)} \mathbf{G}_{2}^{1}(\underline{X})\right] d W\left(\underline{Z}, \underline{Z}^{\dagger}\right) \\
= & \frac{2 n}{\omega_{2 n} R^{n}}(-2 i)^{2 n}(-1)^{\frac{n(n-1)}{2}} \int_{B(\underline{Y}, R)} \mathbf{G}_{2}^{1}(\underline{X}) \widetilde{d V}(\underline{X}) \\
& +\frac{2 R^{2}}{\omega_{2 n} R^{2 n}} \int_{\partial B(\underline{Y}, R)} \widetilde{\mathbf{I}} d \Sigma_{\left(\underline{Z}, \underline{Z}^{\dagger}\right)} \mathcal{D}_{\left(\underline{Z}, \underline{Z}^{\dagger}\right)} \mathbf{G}_{2}^{1}(\underline{X}) \\
= & \frac{2 n}{\omega_{2 n} R^{2 n}}(-2 i)^{n}(-1)^{\frac{n(n-1)}{2}} \int_{B(\underline{Y}, R)} \mathbf{G}_{2}^{1}(\underline{X}) \widetilde{d V}(\underline{X}) .
\end{aligned}
$$

The proof is done.

Corollary 4.8 If the matrix function $\mathbf{G}_{2}^{1}=\left(\begin{array}{l}g_{1}(\underline{X}) g_{2}(\underline{X}) \\ g_{2}(\underline{X}) \\ g_{1}(\underline{\underline{X}})\end{array}\right)$ is $\mathbf{H}$-2-monogenic in $\mathbf{R}^{2 n}$ and satisfies $\left\|\mathbf{G}_{2}^{1}(\underline{X})\right\| \leq M$ for all $\underline{X} \in \mathbf{R}^{2 n}$, then $\mathbf{G}_{2}^{1}(\underline{X})$ must be a constant circulant matrix in $\mathbf{R}^{2 n}$.

Proof The proof is similar to the method in Theorem 3.2.

Suppose $\Omega$ is an open bounded non-empty subset of $\mathbf{R}^{2 n}$ with a Liapunov boundary $\partial \Omega$, we usually write $\Omega^{+}=\Omega$ and $\Omega^{-}=\mathbf{R}^{2 n} \backslash \bar{\Omega}$. The notations $\underline{Y}$ and $\underline{Y} \mid$ will be reserved for Clifford vectors associated to points $\Omega^{+}$, while their Hermitian counterparts are denoted $\underline{V}=\frac{1}{2}(\underline{Y}+i \underline{Y} \mid)$ and $\underline{V}^{\dagger}=-\frac{1}{2}(\underline{Y}-i \underline{Y} \mid)$. By means of the matrix approach sketched above, the following Hermitian Plemelj-Sokhotski formula.

We shall introduce the following matrix operators:

$$
\begin{aligned}
& \mathcal{C}\left[\mathbf{G}_{2}^{1}\right](\underline{Y})=\int_{\partial \Omega} \mathbf{E}(\underline{Z}-\underline{V}) d \Sigma_{\left(\underline{Z}, \underline{Z}^{\dagger}\right)} \mathbf{G}_{2}^{1}(\underline{X}), \quad \underline{Y} \in \Omega^{ \pm}, \\
& \mathcal{H}_{\partial \Omega}\left[\mathbf{G}_{2}^{1}\right](\underline{Y})=(-1)^{\frac{n(n+1)}{2}}\left(-\frac{i}{2}\right)^{n} \int_{\partial \Omega} \mathbf{E}(\underline{Z}-\underline{V}) d \Sigma_{\left(\underline{Z}, \underline{Z}^{\dagger}\right)} \mathbf{G}_{2}^{1}(\underline{X}), \quad \underline{Y} \in \partial \Omega,
\end{aligned}
$$

where $\mathbf{G}_{2}^{1}(\underline{X}) \in \mathbf{C}^{0, \alpha}(\partial \Omega)$.

Lemma 4.9 $[18,21]$ Let $\mathbf{G}_{2}^{1}(\underline{X}) \in \mathbf{C}^{0, \alpha}(\partial \Omega)$. Then the boundary values of the Hermitian Cauchy integral $\mathcal{C}\left[\mathbf{G}_{2}^{1}\right]$ are given by

$$
\begin{aligned}
\mathcal{C}\left[\mathbf{G}_{2}^{1}\right]^{ \pm}(\underline{U}) & =\lim _{\underline{Y} \rightarrow \underline{U} \in \partial \Omega \underline{Y} \in \Omega^{ \pm}} \mathcal{C}\left[\mathbf{G}_{2}^{1}\right](\underline{Y}) \\
& =(-1)^{\frac{n(n+1)}{2}}(2 i)^{n}\left( \pm \frac{1}{2} \mathbf{G}_{2}^{1}(\underline{U})+\mathcal{H}_{\partial \Omega}\left[\mathbf{G}_{2}^{1}\right](\underline{U})\right) .
\end{aligned}
$$

Theorem 4.10 Let $B(\underline{a}, R)$ be an open ball centered at a, with radius $R$ in $\mathbf{R}^{2 n}, g_{1}, g_{2} \in$ $C^{1}\left(\mathbf{R}^{2 n} \backslash \partial B(\underline{a}, R), \mathbb{C}_{2 n}\right), \mathcal{D}_{\left(\underline{Z}, \underline{Z}^{\dagger}\right)} \mathbf{G}_{2}^{1}=0$ in $\mathbf{R}^{2 n} \backslash \partial B(\underline{a}, R),\left[\mathbf{G}_{2}^{1}\right]^{+}(\underline{Y})=\left[\mathbf{G}_{2}^{1}\right]^{-}(\underline{Y}) \in \mathbf{C}^{0, \alpha}(\partial B(\underline{a}$, $R)), 0<\alpha \leq 1$. Then $\mathcal{D}_{\left(\underline{Z}, \underline{Z}^{\dagger}\right)} \mathbf{G}_{2}^{1}=0$ in $\mathbf{R}^{2 n}$.

Proof We only need to prove that for any $\underline{Y}_{0} \in \partial B(\underline{a}, R), \mathcal{D}_{\left(\underline{Z}, \underline{Z}^{\dagger}\right)} \mathbf{G}_{2}^{1}\left(\underline{Y}_{0}\right)=0$. Define $\mathbf{G}_{2}^{1}(\underline{Y})=$ $\left[\mathbf{G}_{2}^{1}\right]^{+}(\underline{Y})=\left[\mathbf{G}_{2}^{1}\right]^{-}(\underline{Y}), \underline{Y} \in \partial B(\underline{a}, R)$. For any $\underline{Y}_{0} \in \partial B(\underline{a}, R)$, taking constants $\delta>0, B\left(\underline{Y}_{0}, \delta\right)$ is a ball with the center at $\underline{Y}_{0}$ and radius $\delta$ such that $B(\underline{a}, R) \subset B\left(\underline{Y}_{0}, \delta\right)$. Obviously, $\partial B(\underline{a}, R) \cup$ $\partial B\left(\underline{Y}_{0}, \delta\right)$ is a Liapunov boundary. Using the Hermitian Borel-Pompeiu formula, we have

$$
(-1)^{\frac{n(n+1)}{2}}(2 i)^{n} \mathbf{G}_{2}^{1}\left(\underline{Y}_{1}\right)=\int_{\partial B(\underline{a}, R)} \mathbf{E}\left(\underline{Z}-\underline{V}_{1}\right) d \Sigma_{\left(\underline{Z}, \underline{Z}^{\dagger}\right)} \mathbf{G}_{2}^{1}(\underline{X}), \quad \underline{Y}_{1} \in B(\underline{a}, R),
$$




$$
\begin{aligned}
& (-1)^{\frac{n(n+1)}{2}}(2 i)^{n} \mathbf{G}_{2}^{1}\left(\underline{Y}_{2}\right)=\int_{\partial B(\underline{a}, R) \cup \partial B\left(\underline{Y}_{0}, \delta\right)} \mathbf{E}\left(\underline{Z}-\underline{V}_{2}\right) d \Sigma_{\left(\underline{\underline{Z}} \underline{Z}^{\dagger}\right)} \mathbf{G}_{2}^{1}(\underline{X}), \\
& \underline{Y}_{2} \in \stackrel{o}{B}\left(\underline{Y}_{0}, \delta\right) \backslash \overline{B(\underline{a}, R)} .
\end{aligned}
$$

Using Lemma 4.9 , for $\underline{Y}_{0} \in \partial B(\underline{a}, R)$, we obtain

$$
\begin{aligned}
& \mathbf{G}_{2}^{1}\left(\underline{Y}_{0}\right)=\left[\mathbf{G}_{2}^{1}\right]^{+}\left(\underline{Y}_{0}\right)=\mathbf{G}_{2}^{1}\left(\underline{Y}_{0}\right)+\mathcal{H}_{\partial B(\underline{a}, R)}\left[\mathbf{G}_{2}^{1}\right]\left(\underline{Y}_{0}\right), \\
& \mathbf{G}_{2}^{1}\left(\underline{Y}_{0}\right)=\left[\mathbf{G}_{2}^{1}\right]^{-}\left(\underline{Y}_{0}\right)=\left(\frac{1}{2} \mathbf{G}_{2}^{1}\left(\underline{Y}_{0}\right)+\mathcal{H}_{\partial B(\underline{a}, R) \cup \partial B\left(\underline{Y}_{0}, \delta\right)}\left[\mathbf{G}_{2}^{1}\right]\left(\underline{Y}_{0}\right)\right) .
\end{aligned}
$$

Combining (4.26) with (4.27), we get

$$
(-1)^{\frac{n(n+1)}{2}}(2 i)^{n} \mathbf{G}_{2}^{1}\left(\underline{Y}_{0}\right)=\int_{\partial B\left(\underline{Y}_{0}, \delta\right)} \mathbf{E}\left(\underline{Z}-\underline{V}_{0}\right) d \Sigma_{\left(\underline{Z}, \underline{Z}^{\dagger}\right)} \mathbf{G}_{2}^{1}(\underline{X}) .
$$

Therefore $\mathcal{D}_{\left(\underline{Z}, \underline{Z}^{\dagger}\right)} \mathbf{G}_{2}^{1}\left(\underline{Y}_{0}\right)=0$, and the result follows.

Theorem 4.11 Let $B(\underline{a}, R)$ be an open ball centered at a with radius $R$ in $\mathbf{R}^{2 n}, g_{1}, g_{2} \in$ $C^{2}\left(B(\underline{a}, R), \mathbb{C}_{2 n}\right) \cap C^{1}\left(\overline{B(\underline{a}, R)}, \mathbb{C}_{2 n}\right) g_{1}, g_{2} \in C^{2}\left(B^{-}(\underline{a}, R), \mathbb{C}_{2 n}\right) \cap C^{1}\left(\overline{B^{-}(\underline{a}, R)}, \mathbb{C}_{2 n}\right),\left(\mathcal{D}_{\left(\underline{Z}, \underline{Z}^{\dagger}\right)}\right)^{2} \times$ $\mathbf{G}_{2}^{1}=0$ in $\mathbf{R}^{2 n} \backslash \partial B(\underline{a}, R)$, and $\mathbf{G}_{2}^{1}$ satisfies the following conditions:

$$
\left\{\begin{array}{l}
{\left[\mathbf{G}_{2}^{1}\right]^{+}\left(\underline{Y)}=\left[\mathbf{G}_{2}^{1}\right]^{-}(\underline{Y}) \in \mathbf{C}^{0, \alpha}(\partial B(\underline{a}, R)),\right.} \\
{\left[\mathcal{D}_{\left(\underline{Z}, \underline{Z}^{\dagger}\right)} \mathbf{G}_{2}^{1}\right]^{+}(\underline{Y})=\left[\mathcal{D}_{\left(\underline{Z}, \underline{Z}^{\dagger}\right)} \mathbf{G}_{2}^{1}\right]^{-}(\underline{Y}) \in \mathbf{C}^{0, \beta}(\partial B(\underline{a}, R)),}
\end{array}\right.
$$

where $0<\alpha, \beta \leq 1$, then $\left(\mathcal{D}_{\left(\underline{Z}, \underline{Z}^{\dagger}\right)}\right)^{2} \mathbf{G}_{2}^{1}=0$ in $\mathbf{R}^{2 n}$.

Proof In view of the weak singularity of $\frac{1}{\omega_{2 n}(2-2 n)} \frac{4}{|\underline{X}-\underline{Y}|^{2 n-2}}$, combining Theorem 4.6 with Lemma 4.9 , the theorem can be similarly proved similarly to Theorem 4.10 .

Theorem 4.12 Let $g_{1}, g_{2} \in C^{2}\left(B^{-}(\underline{a}, R), \mathbb{C}_{2 n}\right) \cap C^{1}\left(\overline{B^{-}(\underline{a}, R)}, \mathbb{C}_{2 n}\right),\left(\mathcal{D}_{\left(\underline{Z}, \underline{Z}^{\dagger}\right)}\right)^{2} \mathbf{G}_{2}^{1}=0$ in $B^{-}(\underline{a}$, $R)$,

$$
\left\{\begin{array}{l}
{\left[\mathbf{G}_{2}^{1}\right]^{+}(\underline{Y})=\left[\mathbf{G}_{2}^{1}\right]^{-}(\underline{Y}) \in \mathbf{C}^{0, \alpha}(\partial B(\underline{a}, R)),} \\
{\left[\mathcal{D}_{\left(\underline{Z}, \underline{Z}^{\dagger}\right)} \mathbf{G}_{2}^{1}\right]^{+}(\underline{Y})=\left[\mathcal{D}_{\left(\underline{Z}, \underline{Z}^{\dagger}\right)} \mathbf{G}_{2}^{1}\right]^{-}(\underline{Y}) \in \mathbf{C}^{0, \beta}(\partial B(\underline{a}, R)),}
\end{array}\right.
$$

where $0<\alpha, \beta \leq 1,\left\|\mathbf{G}_{2}^{1}(\underline{X})\right\| \leq M(|\underline{X}| \rightarrow \infty)$, then for $\underline{Y} \in B^{-}(\underline{a}, R)$

$$
\begin{aligned}
(-1)^{\frac{n(n+1)}{2}}(2 i)^{n} \mathbf{G}_{2}^{1}(\underline{Y})= & -\int_{\partial \Gamma} \mathbf{E}(\underline{Z}-\underline{V}) d \Sigma_{\left(\underline{Z}, \underline{Z}^{\dagger}\right)} \mathbf{G}_{2}^{1}(\underline{X}) \\
& +\int_{\partial \Gamma} \mathbf{E}_{1}(\underline{Z}-\underline{V}) d \Sigma_{\left(\underline{Z}, \underline{Z}^{\dagger}\right)} \mathcal{D}_{\left(\underline{Z}, \underline{Z}^{\dagger}\right)} \mathbf{G}_{2}^{1}(\underline{X})+\mathbf{C}_{2 \infty}^{1},
\end{aligned}
$$

where $\mathbf{C}_{2 \infty}^{\mathbf{1}}$ be a constant circulant matrix.

\section{Riemann boundary value problem for $\mathbf{H}$-monogenic functions}

An $R_{0}$ Riemann boundary value problem for $\mathbf{H}$-monogenic functions is denoted as follows:

$$
\begin{cases}\mathcal{D}_{\left(\underline{V}, \underline{\underline{V}}^{\dagger}\right)} \mathbf{G}_{2}^{1}=0, & \text { in } \mathbf{R}^{2 n} \backslash \partial B(\underline{a}, R), \\ {\left[\mathbf{G}_{2}^{1}\right]^{+}\left(\underline{Y)}=\left[\mathbf{G}_{2}^{1}\right]^{-}(\underline{Y}) \mathbf{A}_{2}^{1}+\mathbf{F}_{2}^{1}(\underline{Y}),\right.} & \underline{Y} \in \partial B(\underline{a}, R), \\ \left\|\mathbf{G}_{2}^{1}(\infty)\right\| \leq M, & \end{cases}
$$


where $\mathbf{A}_{2}^{1}$ is any invertible constant circulant matrix, we denote by $\left[\mathbf{A}_{2}^{1}\right]^{-1}$ an invertible element for $\mathbf{A}_{2}^{1}$. Here $\mathbf{F}_{2}^{1}$ is a given circulant matrix function in $\mathbf{C}^{0, \alpha}(\partial B(\underline{a}, R)), 0<\alpha \leq 1$.

Theorem 5.1 The Riemann boundary value problem (5.1) is solvable and the solution can be written as

$$
(-1)^{\frac{n(n+1)}{2}}(2 i)^{n} \mathbf{G}_{2}^{1}(\underline{Y})= \begin{cases}\int_{\partial B(\underline{a}, R)} \mathbf{E}(\underline{Z}-\underline{V}) d \Sigma_{\left(\underline{Z}, \underline{Z}^{\dagger}\right)} \mathbf{F}_{2}^{1}(\underline{X}) & \\ +\mathbf{C}_{2 \infty}^{1}, & \underline{Y} \in B^{+}(\underline{a}, R), \\ \int_{\partial B(\underline{a}, R)} \mathbf{E}(\underline{Z}-\underline{V}) d \Sigma_{\left(\underline{Z}, \underline{Z}^{\dagger}\right)} \mathbf{F}_{2}^{1}(\underline{X})\left[\mathbf{A}_{2}^{1}\right]^{-1} & \\ \quad+\mathbf{C}_{2 \infty}^{1}\left[\mathbf{A}_{2}^{1}\right]^{-1}, & \underline{Y} \in B^{-}(\underline{a}, R) .\end{cases}
$$

Proof Let

$$
\mathcal{X}_{2}^{1}(\underline{Y})= \begin{cases}(-1)^{\frac{n(n+1)}{2}}\left(-\frac{i}{2}\right)^{n} \mathbf{I}, & \underline{Y} \in B^{+}(\underline{a}, R), \\ (-1)^{\frac{n(n+1)}{2}}\left(-\frac{i}{2}\right)^{n}\left[\mathbf{A}_{2}^{1}\right]^{-1}, & \underline{Y} \in B^{-}(\underline{a}, R) .\end{cases}
$$

Furthermore, we denote

$$
\left[\mathcal{X}_{2}^{1}\right]^{-1}(\underline{Y})= \begin{cases}(-1)^{\frac{n(n+1)}{2}}(2 i)^{n} \mathbf{I}, & \underline{Y} \in B^{+}(\underline{a}, R), \\ (-1)^{\frac{n(n+1)}{2}}(2 i)^{n} \mathbf{A}_{2}^{1}, & \underline{Y} \in B^{+}(\underline{a}, R),\end{cases}
$$

and we then have $\mathcal{D}_{\left(\underline{V}, \underline{V}^{\dagger}\right)}\left[\mathcal{X}_{2}^{1}\right]^{-1}(\underline{Y})=0, \underline{Y} \in \mathbf{R}^{2 n} \backslash \partial B(\underline{a}, R)$. The transmission condition

$$
\left[\mathbf{G}_{2}^{1}\right]^{+}\left(\underline{Y)}=\left[\mathbf{G}_{2}^{1}\right]^{-}(\underline{Y}) \mathbf{A}_{2}^{1}+\mathbf{F}_{2}^{1}(\underline{Y)}\right.
$$

can be changed into

$$
\left[\mathbf{G}_{2}^{1}\right]^{+}(\underline{Y})\left[\left[\mathcal{X}_{2}^{1}\right]^{-1}\right]^{+}\left(\underline{Y)}=\left[\mathbf{G}_{2}^{1}\right]^{-}(\underline{Y})\left[\left[\mathcal{X}_{2}^{1}\right]^{-1}\right]^{-}(\underline{Y})+\mathbf{F}_{2}^{1}(\underline{Y})\left[\left[\mathcal{X}_{2}^{1}\right]^{-1}\right]^{+}(\underline{Y}),\right.
$$

and if we denote

$$
\Psi_{2}^{1}(\underline{Y})=\int_{\partial B(\underline{a}, R)} \mathbf{E}(\underline{Z}-\underline{V}) d \Sigma_{\left(\underline{Z}, \underline{Z}^{\dagger}\right)} \mathbf{F}_{2}^{1}(\underline{X}), \quad \underline{Y} \in \mathbf{R}^{2 n} \backslash \partial B(\underline{a}, R),
$$

then $\mathcal{D}_{\left(\underline{\underline{V}}, \underline{V}^{\dagger}\right)} \Psi_{2}^{1}(\underline{Y})=0, \underline{Y} \in \mathbf{R}^{2 n} \backslash \partial B(\underline{a}, R)$, and $\Psi_{2}^{1}(\infty)=\mathbf{O}$. Using Lemma 4.9, we have

$$
\left[\Psi_{2}^{1}\right]^{+}(\underline{Y})-\left[\Psi_{2}^{1}\right]^{-}(\underline{Y})=\mathbf{F}_{2}^{1}(\underline{Y})\left[\left[\mathcal{X}_{2}^{1}\right]^{-1}\right]^{+}(\underline{Y}), \quad \underline{Y} \in \partial B(\underline{a}, R) .
$$

From (5.5) and (5.7) we have

$$
\left[\mathbf{G}_{2}^{1}\left[\mathcal{X}_{2}^{1}\right]^{-1}-\Psi_{2}^{1}\right]^{+}(\underline{Y})=\left[\mathbf{G}_{2}^{1}\left[\mathcal{X}_{2}^{1}\right]^{-1}-\Psi_{2}^{1}\right]^{-}(\underline{Y}), \quad \underline{Y} \in \partial B(\underline{a}, R) .
$$

Combining Theorem 3.2 with Theorem 4.10, there exists a constant $(2 \times 2)$ circulant matrix $\mathbf{C}_{2 \infty}^{\mathbf{1}}$ such that $\left[\mathbf{G}_{2}^{1}\left[\mathcal{X}_{2}^{1}\right]^{-1}-\Psi_{2}^{1}\right](\underline{Y})=\mathbf{C}_{2 \infty}^{\mathbf{1}}$.

On the other hand, it can be directly proved that (5.2) is the solution of (5.1), and the proof is done.

Remark 5.2 If (5.1) is solved in $R_{-1}$, i.e. $\left\|\mathbf{G}_{2}^{1}(\infty)\right\|=0$ is required, then the problem has the unique solution (5.2) (taking $\mathbf{C}_{2 \infty}^{\mathbf{1}}=\mathbf{0}$ ). 


\section{Riemann boundary value problem for H-2-monogenic function in Hermitian Clifford analysis}

In this section, we shall consider the following $R_{0}$ Riemann boundary value problem:

$$
\begin{cases}\left(\mathcal{D}_{\left(\underline{V}, \underline{V}^{\dagger}\right)}\right)^{2} \mathbf{G}_{2}^{1}=0, & \text { in } \mathbf{R}^{2 n} \backslash \partial B(\underline{a}, R), \\ {\left[\mathbf{G}_{2}^{1}\right]^{+}(\underline{Y})=\left[\mathbf{G}_{2}^{1}\right]^{-}(\underline{Y}) \mathbf{A}_{2}^{1}+\mathbf{F}_{2}^{1}(\underline{Y}),} & \underline{Y} \in \partial B(\underline{a}, R), \\ {\left[\mathcal{D}_{\left(\underline{V}, \underline{V}^{\dagger}\right)} \mathbf{G}_{2}^{1}\right]^{+}(\underline{Y})=\left[\mathcal{D}_{\left(\underline{V}, \underline{V}^{\dagger}\right)} \mathbf{G}_{2}^{1}\right]^{-}(\underline{Y}) \mathbf{B}_{2}^{1}+\mathbf{U}_{2}^{1}(\underline{Y}),} & \underline{Y} \in \partial B(\underline{a}, R), \\ \left\|\mathbf{G}_{2}^{1}(\infty)\right\| \leq M, & \end{cases}
$$

where $\mathbf{A}_{2}^{1}, \mathbf{B}_{2}^{1}$ are invertible constant circulant matrices and $\mathbf{F}_{2}^{1}(\underline{Y}), \mathbf{U}_{2}^{1}(\underline{Y})$ are given circulant matrix functions in $\mathbf{C}^{0, \alpha}(\partial B(\underline{a}, R)), 0<\alpha \leq 1$. We shall give the explicit expression of solutions for (6.1).

Theorem 6.1 The Riemann boundary value problem (6.1) is solvable and the solution is given by

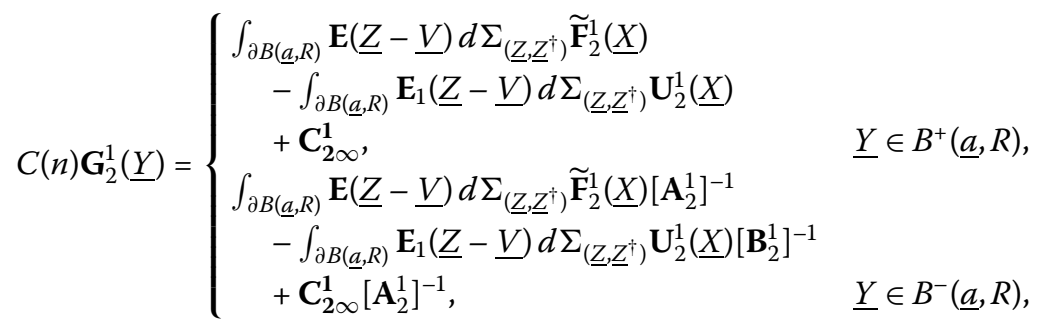

where

$$
\begin{aligned}
C(n)= & (-1)^{\frac{n(n+1)}{2}}(2 i)^{n}, \\
\widetilde{\mathbf{F}}_{2}^{1}(\underline{Y})= & \mathbf{F}_{2}^{1}(\underline{Y})-(-1)^{\frac{n(n+1)}{2}}\left(-\frac{i}{2}\right)^{n} \int_{\partial B(\underline{a}, R)} \mathbf{E}_{1}(\underline{Z}-\underline{V}) d \Sigma_{\left(\underline{Z}, \underline{Z}^{\dagger}\right)} \mathbf{U}_{2}^{1}(\underline{X}) \\
& +(-1)^{\frac{n(n+1)}{2}}\left(-\frac{i}{2}\right)^{n} \int_{\partial B(\underline{a}, R)} \mathbf{E}_{1}(\underline{Z}-\underline{V}) d \Sigma_{\left(\underline{Z}, \underline{Z}^{\dagger}\right)} \mathbf{U}_{2}^{1}(\underline{X})\left[\mathbf{B}_{2}^{1}\right]^{-1} \mathbf{A}_{2}^{1}, \\
& \underline{Y} \in \partial B(\underline{a}, R) .
\end{aligned}
$$

Proof Let $\mathbf{G}_{2}^{1}(\underline{Y})$ be the solution of (6.1) for $\underline{Y} \in \mathbf{R}^{2 n} \backslash \partial B(\underline{a}, R)$. We denote $\mathbf{W}_{2}^{1}(\underline{Y})=$ $\mathcal{D}_{\left(\underline{V}, \underline{V}^{\dagger}\right)} \mathbf{G}_{2}^{1}(\underline{Y})$. Then

$$
\left[\mathbf{W}_{2}^{1}\right]^{+}(\underline{Y})=\left[\mathbf{W}_{2}^{1}\right]^{-}(\underline{Y}) \mathbf{B}_{2}^{1}+\mathbf{U}_{2}^{1}(\underline{Y}), \quad \underline{Y} \in \partial B(\underline{a}, R) .
$$

By $\mathcal{D}_{\left(\underline{V}, \underline{V}^{\dagger}\right)} \mathbf{G}_{2}^{1}(\infty)=\mathbf{O}$ and Theorem 5.1, we have

$$
C(n) \mathbf{W}_{2}^{1}(\underline{Y})= \begin{cases}\int_{\partial B(\underline{a}, R)} \mathbf{E}(\underline{Z}-\underline{V}) d \Sigma_{\left(\underline{Z}, \underline{Z}^{\dagger}\right)} \mathbf{U}_{2}^{1}(\underline{X),} & \underline{Y} \in B^{+}(\underline{a}, R), \\ \int_{\partial B(\underline{a}, R)} \mathbf{E}(\underline{Z}-\underline{V}) d \Sigma_{\left(\underline{Z}, \underline{Z}^{\dagger}\right)} \mathbf{U}_{2}^{1}\left(\underline{X)}\left[\mathbf{B}_{2}^{1}\right]^{-1},\right. & \underline{Y} \in B^{-}(\underline{a}, R) .\end{cases}
$$

We denote

$$
C(n) \mathbf{J}_{2}^{1}(\underline{Y})= \begin{cases}-\int_{\partial B(\underline{a}, R)} \mathbf{E}_{1}(\underline{Z}-\underline{V}) d \Sigma_{\left(\underline{Z}, \underline{Z}^{\dagger}\right.} \mathbf{U}_{2}^{1}(\underline{X}), & \underline{Y} \in B^{+}(\underline{a}, R), \\ -\int_{\partial B(\underline{a}, R)} \mathbf{E}_{1}(\underline{Z}-\underline{V}) d \Sigma_{\left(\underline{Z}, \underline{Z}^{\dagger}\right)} \mathbf{U}_{2}^{1}(\underline{X})\left[\mathbf{B}_{2}^{1}\right]^{-1}, & \underline{Y} \in B^{+}(\underline{a}, R) .\end{cases}
$$


Combining (6.6) with (6.7) we then get

$$
\mathcal{D}_{\left(\underline{V}, \underline{V}^{\dagger}\right)}\left[\mathbf{G}_{2}^{1}-\mathbf{J}_{2}^{1}\right](\underline{Y})=0, \quad \underline{Y} \in \mathbf{R}^{2 n} \backslash \partial B(\underline{a}, R) .
$$

If we denote $\mathbf{G}_{2}^{1}-\mathbf{J}_{2}^{1}:=\boldsymbol{\Phi}_{2}^{1}(\underline{Y})$, where $\underline{Y} \in \mathbf{R}^{2 n} \backslash \partial B(\underline{a}, R)$ and use

$$
\left[\mathbf{G}_{2}^{1}\right]^{+}(\underline{Y})=\left[\mathbf{G}_{2}^{1}\right]^{-}(\underline{Y}) \mathbf{A}_{2}^{1}+\mathbf{F}_{2}^{1}(\underline{Y}), \quad \underline{Y} \in \partial B(\underline{a}, R),
$$

then we obtain

$$
\left[\boldsymbol{\Phi}_{2}^{1}\right]^{+}(\underline{Y})=\left[\boldsymbol{\Phi}_{2}^{1}\right]^{-}(\underline{Y}) \mathbf{A}_{2}^{1}+\widetilde{\mathbf{F}}_{2}^{1}(\underline{Y}), \quad \underline{Y} \in \partial B(\underline{a}, R)
$$

where $\widetilde{\mathbf{F}}_{2}^{1}(\underline{Y})$ is denoted as in (6.4).

It is obvious that $\widetilde{\mathbf{F}}_{2}^{1}(\underline{Y}) \in \mathbf{C}^{0, \alpha}(\partial B(\underline{a}, R)), 0<\alpha \leq 1$. Since $\left\|\boldsymbol{\Phi}_{2}^{1}(\infty)\right\| \leq M$, using Theorem 5.1 we get the following representation:

$$
C(n) \boldsymbol{\Phi}_{2}^{1}\left(\underline{Y)}= \begin{cases}\int_{\partial B(\underline{a}, R)} \mathbf{E}(\underline{Z}-\underline{V}) d \Sigma_{\left(\underline{Z}, \underline{Z}^{\dagger}\right)} \widetilde{\mathbf{F}}_{2}^{1}(\underline{X)} & \\ +\mathbf{C}_{2 \infty}^{1}, & \underline{Y} \in B^{+}(\underline{a}, R), \\ \int_{\partial B(\underline{a}, R)} \mathbf{E}(\underline{Z}-\underline{V}) d \Sigma_{\left(\underline{Z}, \underline{Z}^{\dagger}\right)} \widetilde{\mathbf{F}}_{2}^{1}\left(\underline{X)}\left[\mathbf{A}_{2}^{1}\right]^{-1}\right. & \\ +\mathbf{C}_{2 \infty}^{1}\left[\mathbf{A}_{2}^{1}\right]^{-1}, & \underline{Y} \in B^{-}(\underline{a}, R) .\end{cases}\right.
$$

Combining (6.7) with (6.10) we arrive at the proposed result.

On the other hand, it can be directly proved that (6.2) are the solution of (6.1) and the proof is done.

Remark 6.2 If (6.1) is solved in $R_{-1}$, i.e. $\left\|\mathbf{G}_{2}^{1}(\infty)\right\|=0$ is required, then the problem has the unique solution (6.2) (taking $\mathbf{C}_{2 \infty}^{\mathbf{1}}=\mathbf{0}$ ).

\section{Competing interests}

The authors declare that they have no competing interests.

\section{Authors' contributions}

All authors contributed equally to the manuscript and typed, read, and approved the final manuscript.

\section{Acknowledgements}

This paper is supported by National Natural Science Foundation of China (11271175), the AMEP, and DYSP of Linyi University. The authors would like to thank the referees for their valuable suggestion and comments.

Received: 3 November 2013 Accepted: 28 March 2014 Published: 09 Apr 2014

\section{References}

1. Muskhelishvilli, Nl: Singular Integral Equations. Nauka, Moscow (1968)

2. Lu, J: Boundary Value Problems of Analytic Functions. World Scientific, Singapore (1993)

3. Brackx, F, Delanghe, R, Sommen, F: Clifford Analysis. Research Notes in Mathematics, vol. 76. Pitman, London (1982)

4. Delanghe, R, Sommen, F, Souček, V: Clifford Algebra and Spinor-Valued Functions. Kluwer Academic, Dordrecht (1992)

5. Delanghe, R: On the regular analytic functions with values in a Clifford algebra. Math. Ann. 185, $91-111$ (1970)

6. Delanghe, R: On the singularities of functions with values in a Clifford algebra. Math. Ann. 196, 293-319 (1972)

7. Bernstein, S: On the left linear Riemann problem in Clifford analysis. Bull. Belg. Math. Soc. Simon Stevin 3, 557-576 (1996)

8. Zhang, Z, Du, J: On certain Riemann boundary value problems and singular integral equations in Clifford analysis. Chin. Ann. Math., Ser. A 22, 421-426 (2000)

9. Xu, Z: On linear and nonlinear Riemann-Hilbert problems for regular functions with values in a Clifford algebra. Chin. Ann. Math., Ser. B 11(3), 349-358 (1990) 
10. Abreu Blaya, R, Bory Reyes, J: On the Riemann Hilbert type problems in Clifford analysis. Adv. Appl. Clifford Algebras 11(1), 15-26 (2001)

11. Bory Reyes, J, Abreu Blaya, R: The quaternionic Riemann problem with natural geometric condition on the boundary. Complex Var. Theory Appl. 42, 135-149 (2000)

12. Abreu Blaya, R, Bory Reyes, J: Boundary value problems for quaternionic monogenic functions on non-smooth surfaces. Adv. Appl. Clifford Algebras 9(1), 1-22 (1999)

13. Gürlebeck, K, Zhang, Z: Some Riemann boundary value problems in Clifford analysis. Math. Methods Appl. Sci. 33 287-302 (2010)

14. Zhang, Z, Gürlebeck, K: Some Riemann boundary value problems in Clifford analysis (I). Complex Var. Elliptic Equ. 58, 991-1003 (2013)

15. Brackx, F, Bureš, J, De Schepper, H, Eelbode, D, Sommen, F, Soucěk, V: Fundaments of Hermitean Clifford analysis part I: complex structure. Complex Anal. Oper. Theory 1(3), 341-365 (2007)

16. Brackx, F, Bureš, J, De Schepper, H, Eelbode, D, Sommen, F, Soucěk, V: Fundamentals of Hermitean Clifford analysis part II: splitting of $h$-monogenic equations. Complex Var. Elliptic Equ. 52, 1068-1078 (2007)

17. Brackx, F, De Knock, B, De Schepper, H, Sommen, F: On Cauchy and Martinelli-Bochner integral formulae in Hermitean Clifford analysis. Bull. Braz. Math. Soc. 40(3), 395-461 (2009)

18. Brackx, F, De Knock, B, De Schepper, H: A matrix Hilbert transform in Hermitean Clifford analysis. J. Math. Anal. Appl. 344, 1068-1078 (2008)

19. Kytmanov, AM: The Bochner-Martinelli Integral and Its Applications. Brikhäuser, Basel (1995)

20. Rocha-Chavez, R, Shapiro, M, Sommen, F: Integral Theorem for Functions and Differential Forms in $\mathbb{C}_{m}$. Res. Notes Math., vol. 428. Chapman \& Hall/CRC, New York (2002)

21. Abreu Blaya, R, Bory Reyes, J, Brackx, F, De Knock, B, De Schepper, H, Peña Peña, D, Sommen, F: Hermitean Cauchy integral decomposition of continuous functions on hypersurfaces. Bound. Value Probl. 2008, Article ID 425256 (2008)

22. Abreu Blaya, R, Bory Reyes, J, Brackx, F, De Schepper, H, Sommen, F: Boundary value problems associated to a Hermitian Helmholtz equation. J. Math. Anal. Appl. 389, 1268-1279 (2012)

23. Gürlebeck, K, Sprössig, W: Quaternionic and Clifford Calculus for Physicists and Engineers. Wiley, Chichester (1997)

24. Gürlebeck, K, Habetha, K Sprössig, W: Holomorphic Functions in the Plane and n-Dimensional Space. Birkhäuser, Basel (2008)

25. Kravchenko, WV, Shapiro, MV: Integral Representations for Spatial Models of Mathematical Physics. Pitman Research in Mathematics Series, vol. 351. Longman, Harlow (1996)

10.1186/1687-2770-2014-81

Cite this article as: Gu and Fu: Riemann boundary value problem for $\mathbf{H}$-2-monogenic function in Hermitian Clifford analysis. Boundary Value Problems 2014, 2014:81

\section{Submit your manuscript to a SpringerOpen ${ }^{\ominus}$ journal and benefit from:}

- Convenient online submission

- Rigorous peer review

- Immediate publication on acceptance

- Open access: articles freely available online

- High visibility within the field

- Retaining the copyright to your article 